\title{
Twisted Rota-Baxter operators and Reynolds operators on Lie algebras and NS-Lie algebras
}

\author{
Apurba Das \\ Department of Mathematics and Statistics, \\ Indian Institute of Technology, Kanpur 208016, Uttar Pradesh, India. \\ Email: apurbadas348@gmail.com
}

\begin{abstract}
In this paper, we introduce twisted Rota-Baxter operators on Lie algebras as an operator analogue of twisted $r$-matrices. We construct a suitable $L_{\infty}$-algebra whose Maurer-Cartan elements are given by twisted Rota-Baxter operators. This allows us to define cohomology of a twisted Rota-Baxter operator. This cohomology can be seen as the Chevalley-Eilenberg cohomology of a certain Lie algebra with coefficients in a suitable representation. We study deformations of twisted Rota-Baxter operators from cohomological points of view. Some applications are given to Reynolds operators and twisted $r$-matrices. Next, we introduce a new algebraic structure, called NS-Lie algebras, that is related to twisted Rota-Baxter operators in the same way pre-Lie algebras are related to Rota-Baxter operators. We end this paper by considering twisted generalized complex structures on modules over Lie algebras.
\end{abstract}

2010 MSC classification: 17B56, 16T25, 17B37, 17B40.

Keywords: Twisted Rota-Baxter operators, Reynolds operators, Cohomology, Deformations, NS-Lie algebras.

\section{Introduction}

Rota-Baxter operators and more generally $\mathcal{O}$-operators on Lie algebras was first appeared in a paper of B. A. Kupershmidt [15] as an operator analogue of classical $r$-matrices and Poisson structures. However, Rota-Baxter operators have origin in the work of G.-C. Rota [22] and G. Baxter [1] in fluctuation theory of probability and combinatorics. They have important applications in the algebraic aspects of the renormalization in quantum field theory [3]. Rota-Baxter operators on Lie algebras (resp. associative algebras) are also related to the splitting of algebras, specifically with pre-Lie algebras (resp. dendriform algebras). Recently, deformations of Rota-Baxter operators on Lie algebras has been carried out in [18,24], and has been extended to the case of associative algebras in [6].

The notion of twisted Poisson structures was introduced by P. Ševera and A. Weinstein [23] who showed that twisted Poisson structures can be described by Dirac structures in a Courant algebroid. C. Klimčík and T. Strobl [12] also studied twisted Poisson structures from geometric points of view. Motivated by this, K. Uchino [25] defined a notion of twisted Rota-Baxter operator in the context of associative algebras as an operator analogue twisted Poisson structures and find relations with NS-algebras of P. Leroux [19].

Our primary aim in this paper is to introduce and study twisted Rota-Baxter operators on Lie algebras. Let $H$ be a 2-cocycle in the Chevally-Eilenberg complex of a Lie algebra $\mathfrak{g}$ with coefficients in a representation $M$. A $H$-twisted Rota-Baxter operator is a linear map $T: M \rightarrow \mathfrak{g}$ that satisfies the Rota-Baxter type identity modified by the 2-cocycle $H$ (see Definition 2.1). The inverse of a 1-cochain $h: \mathfrak{g} \rightarrow M$ (when $h$ is invertible) is a $H$-twisted Rota-Baxter operator with $H=-\delta_{\mathrm{CE}} h$. Here $\delta_{\mathrm{CE}}$ is the Chevalley-Eilenberg 
differential. If $r \in \wedge^{2} \mathfrak{g}$ is a twisted $r$-matrix, then $r^{\sharp}: \mathfrak{g}^{*} \rightarrow \mathfrak{g}$ is a twisted Rota-Baxter operator (see Example 2.10). We provide some other examples including the one that arises from a Nijenhuis operator on a Lie algebra. Particular classes of twisted Rota-Baxter operators are given by Reynolds operators, which were first introduced in the associative algebra context (see [27] for the literature). Reynolds operators are shown to be closely related with derivations on the Lie algebra.

In [24] the authors construct a graded Lie algebra using Voronov's derived bracket [26], whose MaurerCartan elements are Rota-Baxter operators on Lie algebras. We introduce a ternary bracket (associated to a 2-cocycle $H$ ) on the underlying graded vector space which makes it an $L_{\infty}$-algebra. The MaurerCartan elements of this $L_{\infty}$-algebra are precisely $H$-twisted Rota-Baxter operators. This allows us to define cohomology of a $H$-twisted Rota-Baxter operator. On the other hand, given a $H$-twisted RotaBaxter operator, we construct a Lie algebra structure on $M$ and a representation of it on the vector space $\mathfrak{g}$. The corresponding Chevalley-Eilenberg cohomology is shown to be isomorphic with the cohomology of $T$.

The classical formal deformation theory of Gerstenhaber [8] has been extended to Rota-Baxter operators on Lie algebras in [24]. We apply the same approach to $H$-twisted Rota-Baxter operators. We show that the infinitesimal in a deformation of $T$ is a 1-cocycle in the cohomology of $T$. Moreover, we define a notion of equivalence between two formal deformations of $T$. The infinitesimals corresponding to equivalent deformations are shown to be cohomologous. We introduce Nijenhuis elements associated with a $H$-twisted Rota-Baxter operator. Such Nijenhuis elements are obtained from trivial linear deformations. We also find a sufficient condition for the rigidity of a $H$-twisted Rota-Baxter operator in terms of Nijenhuis elements.

In the next, we introduce a new algebraic structure consisting of two binary operations, the second of which is skew-symmetric and these two operations are supposed to satisfy two new identities. We call such algebras as NS-Lie algebras. They are a generalization of pre-Lie algebras and a skew-symmetric analogue of (associative) NS-algebras introduced by Leroux [19]. An NS-Lie algebra naturally associates a Lie algebra structure, called the adjacent Lie algebra. We give some examples of NS-Lie algebras, specially obtained from Nijenhuis operators, (associative) NS-algebras and $H$-twisted Rota-Baxter operators. We show the category of twisted Rota-Baxter operators and the category of NS-Lie algebras are equivalent. More details study of NS-Lie algebras and their adjacent Lie algebras will be treated in a subsequent paper.

Finally, inspired by the generalized complex structures $[10,11]$ on differential geometry, we introduce $H$ twisted generalized complex structures on modules over Lie algebras. Such a structure has an underlying Rota-Baxter operator. Motivated from the explicit description of a generalized complex structure, we obtain the explicit form of a twisted generalized complex structure on a module over a Lie algebra. Some examples are also given.

The paper is organized as follows. In the next section (section 2), we introduce $H$-twisted Rota-Baxter operators and Reynolds operators on Lie algebras. In section 3 , we consider an $L_{\infty}$-algebra whose MaurerCartan elements are given by $H$-twisted Rota-Baxter operators. This motivates us to define cohomology of a $H$-twisted Rota-Baxter operator $T: M \rightarrow \mathfrak{g}$. We show that a $H$-twisted Rota-Baxter operator $T$ induces a Lie algebra structure on $M$ which has representation on $\mathfrak{g}$. The corresponding Chevalley-Eilenberg cohomology is shown to be isomorphic with the cohomology of $T$. Deformations of $H$-twisted Rota-Baxter operators from cohomological points of view has been treated in section 4. Some applications to Reynolds operators and twisted $r$-matrices are given in section 5. We introduce NS-Lie algebras and obtain their relation with Nijenhuis operators, (associative) NS-algebras and $H$-twisted Rota-Baxter operators in section 6. Finally, in section 7 , we consider twisted generalized complex structures on modules over Lie algebras.

All (graded) vector spaces, linear maps, tensor products and wedge products are over a field $\mathbb{K}$ of characteristic zero. 


\section{Twisted Rota-Baxter operators and Reynolds operators on Lie algebras}

Let $(\mathfrak{g},[]$,$) be a Lie algebra. A representation of \mathfrak{g}$ consists of a vector space $M$ together with a bilinear map $\bullet: \mathfrak{g} \times M \rightarrow M,(x, m) \mapsto x \bullet m$ satisfying the following

$$
[x, y] \bullet u=x \bullet(y \bullet u)-y \bullet(x \bullet u), \text { for } x, y \in \mathfrak{g}, u \in M
$$

Given a Lie algebra $\mathfrak{g}$ and a representation $M$, the Chevalley-Eilenberg cohomology of $\mathfrak{g}$ with coefficients in $M$ is given by the cohomology of the cochain complex $\left(\left\{C_{\mathrm{CE}}^{n}(\mathfrak{g}, M)\right\}_{n \geq 0}, \delta_{\mathrm{CE}}\right)$, where $C_{\mathrm{CE}}^{n}(\mathfrak{g}, M)=$ $\operatorname{Hom}\left(\wedge^{n} \mathfrak{g}, M\right)$, for $n \geq 0$ and the differential $\delta_{\mathrm{CE}}: C_{\mathrm{CE}}^{n}(\mathfrak{g}, M) \rightarrow C_{\mathrm{CE}}^{n+1}(\mathfrak{g}, M)$ given by

$$
\begin{aligned}
\left(\delta_{\mathrm{CE}} f\right)\left(x_{1}, \ldots, x_{n+1}\right)= & \sum_{i=1}^{n+1}(-1)^{i+1} x_{i} \bullet f\left(x_{1}, \ldots, \widehat{x_{i}}, \ldots, x_{n+1}\right) \\
& +\sum_{1 \leq i<j \leq n+1}(-1)^{i+j} f\left(\left[x_{i}, x_{j}\right], x_{1}, \ldots, \widehat{x_{i}}, \ldots, \widehat{x_{j}}, \ldots, x_{n+1}\right) .
\end{aligned}
$$

The corresponding cohomology groups are denoted by $H_{\mathrm{CE}}^{n}(\mathfrak{g}, M)$, for $n \geq 0$.

Let $H \in C_{\mathrm{CE}}^{2}(\mathfrak{g}, M)$ be a 2-cocycle, i.e. $H: \mathfrak{g} \times \mathfrak{g} \rightarrow M$ is a skew-symmetric bilinear map satisfing

$$
x \bullet H(y, z)+y \bullet H(z, x)+z \bullet H(x, y)+H(x,[y, z])+H(y,[z, x])+H(z,[x, y])=0, \quad \text { for } x, y, z \in \mathfrak{g} \text {. }
$$

2.1 Definition. A linear map $T: M \rightarrow \mathfrak{g}$ is said to a $H$-twisted Rota-Baxter operator if $T$ satisfies

$$
[T(u), T(v)]=T(T(u) \bullet v-T(v) \bullet u+H(T u, T v)), \text { for } u, v \in M
$$

Let $\mathfrak{g}$ be a Lie algebra and $M$ be a $\mathfrak{g}$-module. For any 2-cocycle $H$, the direct sum $\mathfrak{g} \oplus M$ carries a Lie algebra structure given by

$$
[(x, u),(y, v)]^{H}=([x, y], x \bullet v-y \bullet u+H(x, y))
$$

This is called the $H$-twisted semi-direct product, denoted by $\mathfrak{g} \ltimes_{H} M$.

2.2 Proposition. A linear map $T: M \rightarrow \mathfrak{g}$ is a $H$-twisted Rota-Baxter operator if and only if the graph $\operatorname{Gr}(T)=\{(T u, u) \mid u \in M\}$ is a subalgebra of the $H$-twisted semi-direct product $\mathfrak{g} \ltimes_{H} M$.

2.3 Remark. Rota-Baxter operators on Lie algebras generalize classical r-matrices [15], hence generalize Poisson structures on a manifold. This can be viewed by the following similarities between Rota-Baxter operators and Poisson structures. A linear map $T: M \rightarrow \mathfrak{g}$ is a Rota-Baxter operator if and only if $\operatorname{Gr}(T) \subset \mathfrak{g} \oplus M$ is a subalgebra of the semi-direct product, on the other hand, a bivector field $\pi \in \Gamma\left(\wedge^{2} T M\right)$ on a manifold is a Poisson tensor if and only if the graph $\operatorname{Gr}\left(\pi^{\sharp}\right)$ of the bundle map $\pi^{\sharp}: T^{*} M \rightarrow T M$ is closed under the Courant bracket [, ] $]_{\text {cou }}$ on $T M \oplus T^{*} M$. In [23] Ševera and Weinstein introduced a $H$-twisted Courant bracket [ , ] $]_{\text {cou }}^{H}$ on $T M \oplus T^{*} M$, for any closed 3-form $H \in \Omega_{\text {cl }}^{3}(M)$. A bivector field $\pi$ is called a $H$-twisted Poisson structure if $\operatorname{Gr}\left(\pi^{\sharp}\right)$ is closed under the $H$-twisted Courant bracket [, $]_{\text {Cou }}^{H}$. In this regard, the notion of $H$-twisted Rota-Baxter operators are generalization of $H$-twisted Poisson structures.

The following result is a consequence of Proposition 2.2.

2.4 Proposition. Let $T: M \rightarrow \mathfrak{g}$ be a $H$-twisted Rota-Baxter operator. Then $M$ carries a Lie algebra structure with bracket

$$
[u, v]_{T}:=T(u) \bullet v-T(v) \bullet u+H(T u, T v), \quad \text { for } u, v \in M
$$


2.5 Definition. Let $T: M \rightarrow \mathfrak{g}$ be a $H$-twisted Rota-Baxter operator. Suppose $\mathfrak{g}^{\prime}$ is another Lie algebra, $M^{\prime}$ is a $\mathfrak{g}^{\prime}$-module and $H^{\prime} \in C_{\mathrm{CE}}^{2}\left(\mathfrak{g}^{\prime}, M^{\prime}\right)$ is a 2-cocycle. Let $T^{\prime}: M^{\prime} \rightarrow \mathfrak{g}^{\prime}$ be a $H^{\prime}$-twisted Rota-Baxter operator. A morphism of twisted Rota-Baxter operators from $T$ to $T^{\prime}$ consists of a pair $(\phi, \psi)$ of a Lie algebra morphism $\phi: \mathfrak{g} \rightarrow \mathfrak{g}^{\prime}$ and a linear map $\psi: M \rightarrow M^{\prime}$ satisfying

$$
\psi(x \bullet u)=\phi(x) \bullet \psi(u), \quad \psi \circ H=H^{\prime} \circ(\phi \otimes \phi) \quad \text { and } \quad \phi \circ T=T^{\prime} \circ \psi, \quad \text { for } x \in \mathfrak{g}, u \in M .
$$

2.6 Example. Any Rota-Baxter operator of weight 0 (more generally any $\mathcal{O}$-operator or Kuperschmidt operator) on a Lie algebra is a $H$-twisted Rota-Baxter operator with $H=0$.

2.7 Example. Let $\mathfrak{g}$ be a Lie algebra and $M$ be a $\mathfrak{g}$-module. Suppose $h: \mathfrak{g} \rightarrow M$ is an invertible 1-cochain in the Chevalley-Eilenberg cochain complex of $\mathfrak{g}$ with coefficients in $M$. Then $T=h^{-1}: M \rightarrow \mathfrak{g}$ is a $H$-twisted Rota-Baxter operator with $H=-\delta_{\mathrm{CE}} h$. To verify this, we observe that

$$
H(T u, T v)=-\left(\delta_{\mathrm{CE}} h\right)(T u, T v)=-T(u) \bullet v+T(v) \bullet u+h([T u, T v]) .
$$

By applying $T$ to both sides of (3), we get the identity (1).

2.8 Example. Let $\mathfrak{g}$ be a Lie algebra with Lie bracket given by the map $\mu: \wedge^{2} \mathfrak{g} \rightarrow \mathfrak{g}$. Note that the space $M=\wedge^{2} \mathfrak{g}$ is a $\mathfrak{g}$-module by $[x, y \wedge z]=[x, y] \wedge z+y \wedge[x, z]$, for $x \in \mathfrak{g}$ and $y \wedge z \in \wedge^{2} \mathfrak{g}$. Moreover, the map $H: \wedge^{2} \mathfrak{g} \rightarrow \wedge^{2} \mathfrak{g}, y \wedge z \mapsto-y \wedge z$ is a 2-cocycle in the Chevalley-Eilenberg cohomology of $\mathfrak{g}$ with coefficients in $\wedge^{2} \mathfrak{g}$. With this notation, the map $\mu: \wedge^{2} \mathfrak{g} \rightarrow \mathfrak{g}$ is a $H$-twisted Rota-Baxter operator.

2.9 Example. Let $N: \mathfrak{g} \rightarrow \mathfrak{g}$ be a Nijenhuis operator on a Lie algebra $\mathfrak{g}$, i.e. $N$ satisfies

$$
[N x, N y]=N([N x, y]+[x, N y]-N[x, y]), \quad \text { for } x, y \in \mathfrak{g} .
$$

In this case $\mathfrak{g}$ carries a new Lie bracket $[x, y]_{N}=[N x, y]+[x, N y]-N[x, y]$, for $x, y \in \mathfrak{g}$. We denote this Lie algebra structure by $\mathfrak{g}_{N}$. Moreover, the Lie algebra $\mathfrak{g}_{N}$ has a representation on $\mathfrak{g}$ by $x \bullet y:=[N x, y]$, for $x \in \mathfrak{g}_{N}, y \in \mathfrak{g}$. With this representation, the map $H: \wedge^{2} \mathfrak{g}_{N} \rightarrow \mathfrak{g}, H(x, y)=-N[x, y]$ is a 2-cocycle in the Chevalley-Eilenberg cohomology of $\mathfrak{g}_{N}$ with coefficients in $\mathfrak{g}$. Then it is easy to observe that the identity map id $: \mathfrak{g} \rightarrow \mathfrak{g}_{N}$ is a $H$-twisted Rota-Baxter operator.

This example will be more clear in Section 6 when we will introduce NS-Lie algebras and a functor from the category of NS-Lie algebras to the category of twisted Rota-Baxter operators.

2.10 Example. (Twisted triangular $r$-matrix) Let $\mathfrak{g}$ be a Lie algebra and $\psi \in \wedge^{3} \mathfrak{g}^{*}$ be a 3 -cocycle of $\mathfrak{g}$ with coefficients in $\mathbb{K}$. Then an element $\mathbf{r} \in \wedge^{2} \mathfrak{g}$ is called a $\psi$-twisted triangular $r$-matrix if $\mathbf{r}$ satisfies

$$
\left[\mathbf{r}_{12}, \mathbf{r}_{13}\right]+\left[\mathbf{r}_{12}, \mathbf{r}_{23}\right]+\left[\mathbf{r}_{13}, \mathbf{r}_{23}\right]=-\left(\wedge^{3} \mathbf{r}^{\sharp}\right)(\psi),
$$

where $\mathbf{r}^{\sharp}: \mathfrak{g}^{*} \rightarrow \mathfrak{g}, \alpha \mapsto \mathbf{r}(\alpha$,$) is the map induced by \mathbf{r}$. The equation (4) is called the $\psi$-twisted classical Yang-Baxter equation. Note that twisted $r$-matrices are Lie algebraic version of twisted Poisson structures [23].

Observe that, $\psi \in \wedge^{3} \mathfrak{g}^{*}$ is a 3 -cocycle in the cohomology of $\mathfrak{g}$ with coefficients in $\mathbb{K}$ imples that $\psi^{\sharp}: \wedge^{2} \mathfrak{g} \rightarrow \mathfrak{g}^{*}$ is a 2-cocycle in the cohomology of $\mathfrak{g}$ with coefficients in the coadjoint representation $\mathfrak{g}^{*}$. With this notation, an element $\mathbf{r} \in \wedge^{2} \mathfrak{g}$ is a $\psi$-twisted triangular $r$-matrix if and only if $\mathbf{r}^{\sharp}: \mathfrak{g}^{*} \rightarrow \mathfrak{g}$ is a $\psi^{\sharp}$-twisted Rota-Baxter operator on $\mathfrak{g}^{*}$ over the Lie algebra $\mathfrak{g}$.

Let $\mathbf{r} \in \wedge^{2} \mathfrak{g}$ be a $\psi$-twisted triangular $r$-matrix. Then it has been observed in [14] that $\mathfrak{g}^{*}$ carries a Lie algebra structure with bracket

$$
[\alpha, \beta]_{\mathbf{r}, \psi}:=a d_{\mathbf{r}^{\sharp}(\alpha)}^{*} \beta-a d_{\mathbf{r}^{\sharp}(\beta)}^{*} \alpha+\psi\left(\mathbf{r}^{\sharp} \alpha, \mathbf{r}^{\sharp} \beta,\right), \quad \text { for } \alpha, \beta \in \mathfrak{g}^{*} .
$$

Moreover, the map $\mathbf{r}^{\sharp}: \mathfrak{g}^{*} \rightarrow \mathfrak{g}$ is a Lie algebra morphism, equivalently, $\left[\mathbf{r}^{\sharp} \alpha, \mathbf{r}^{\sharp} \beta\right]=\mathbf{r}^{\sharp}\left([\alpha, \beta]_{\mathbf{r}, \psi}\right)$, for $\alpha, \beta \in \mathfrak{g}^{*}$. 
Given a $H$-twisted Rota-Baxter operator $T$ and a 1 -cochain $h$, here we construct a $(H+\delta h)$-twisted Rota-Baxter operator under certain condition. First we observe the following.

2.11 Proposition. Let $\mathfrak{g}$ be a Lie algebra and $M$ be a $\mathfrak{g}$-module. For any 2 -cocycle $H \in C_{\mathrm{CE}}^{2}(\mathfrak{g}, M)$ and 1-cochain $h \in C_{\mathrm{CE}}^{1}(\mathfrak{g}, M)$, we have isomorphism of Lie algebras

$$
\mathfrak{g} \ltimes_{H} M \cong \mathfrak{g} \ltimes_{H+\delta h} M .
$$

Proof. Define $\Psi_{h}: \mathfrak{g} \ltimes_{H} M \rightarrow \mathfrak{g} \ltimes_{H+\delta h} M$ by $\Psi_{h}(x, u)=(x, u-h(x))$, for $(x, u) \in \mathfrak{g} \oplus M$. Then we have

$$
\begin{aligned}
\Psi_{h}[(x, u),(y, v)]^{H} & =([x, y], x \bullet v-y \bullet u+H(x, y)-h[x, y]) \\
& =([x, y], x \bullet v-y \bullet u+H(x, y)-x \bullet h(y)+y \bullet h(x)+(\delta h)(x, y)) \\
& =[(x, u-h(x)),(y, v-h(y))]^{H+\delta h} \\
& =\left[\Psi_{h}(x, u), \Psi_{h}(y, v)\right]^{H+\delta h} .
\end{aligned}
$$

This proves the result.

Let $T: M \rightarrow \mathfrak{g}$ be a $H$-twisted Rota-Baxter operator and $h \in C_{\mathrm{CE}}^{1}(\mathfrak{g}, M)$ be a 1-cochain. Consider the subalgebra $\operatorname{Gr}(T) \subset \mathfrak{g} \ltimes_{H} M$ of the twisted semi-direct product. It follows from Proposition 2.11 that $\Psi_{h}(\operatorname{Gr}(T))=\{(T u, u-h T(u)) \mid u \in M\} \subset \mathfrak{g} \ltimes_{H+\delta h} M$ is a subalgebra. If the linear map (id $\left.-h \circ T\right): M \rightarrow M$ is invertible, then $\Psi_{h}(\operatorname{Gr}(T))$ is the graph of the linear map $T(\mathrm{id}-h \circ T)^{-1}$. In this case, it follows from Proposition 2.2 that $T(\mathrm{id}-h \circ T)^{-1}$ is a $(H+\delta h)$-twisted Rota-Baxter operator.

In the next, we give a construction of a new $H$-twisted Rota-Baxter operator out of an old one and a suitable 1-cocycle. Let $T: M \rightarrow \mathfrak{g}$ be a $H$-twisted Rota-Baxter operator. Consider the $\operatorname{graph} \operatorname{Gr}(T)=$ $\{(T u, u) \mid u \in M\} \subset \mathfrak{g} \ltimes_{H} M$ which is a subalgebra of the $H$-twisted semi-direct product.

Let $B: \mathfrak{g} \rightarrow M$ be a 1-cocycle in the Chevalley-Eilenberg cohomology of $\mathfrak{g}$ with coefficients in $M$. Since $B$ is a 1-cocycle, the deformed subspace

$$
\tau_{B}(\operatorname{Gr}(T))=\{(T u, u+B T(u)) \mid u \in M\} \subset \mathfrak{g} \ltimes_{H} M
$$

is still a subalgebra. This subalgebra may not be the graph of a linear map from $M$ to $\mathfrak{g}$. However, if the linear map (id $+B \circ T): M \rightarrow M$ is invertible, then $\tau_{B}(\operatorname{Gr}(T)$ ) is the graph of the linear map $T(\mathrm{id}+B \circ T)^{-1}: M \rightarrow \mathfrak{g}$. In this case, we say that $B$ is $T$-admissible 1 -cocycle. Then it follows from Proposition 2.2 that $T(\mathrm{id}+B \circ T)^{-1}$ is a $H$-twisted Rota-Baxter operator. This is called the gauge transformation of $T$ associated with $B$. We denote this $H$-twisted Rota-Baxter operator by $T_{B}$.

2.12 Proposition. Let $T$ be a $H$-twisted Rota-Baxter operator and $B$ be a $T$-admissible 1-cocycle. Then the Lie algebra structures on $M$ induced by $T$ and $T_{B}$ are isomorphic.

Proof. Consider the linear isomorphism id $+B \circ T: M \rightarrow M$. We have

$$
\begin{aligned}
& {[(\mathrm{id}+B \circ T)(u),(\mathrm{id}+B \circ T)(v)]_{T_{B}}} \\
& =T(u) \bullet(\mathrm{id}+B \circ T)(v)-T(v) \bullet(\mathrm{id}+B \circ T)(u)+H(T u, T v) \\
& =T(u) \bullet v-T(v) \bullet u+T(u) \bullet B T(v)-T(v) \bullet B T(u)+H(T u, T v) \\
& =T(u) \bullet v-T(v) \bullet u+B([T u, T v])+H(T u, T v) \\
& =[u, v]_{T}+B T\left([u, v]_{T}\right)=(\mathrm{id}+B \circ T)\left([u, v]_{T}\right) .
\end{aligned}
$$

Hence it follows that $(\mathrm{id}+B \circ T):\left(M,[,]_{T}\right) \rightarrow\left(M,[,]_{T_{B}}\right)$ is a Lie algebra isomorphism. 


\subsection{Reynolds operators}

Given a Lie algebra $(\mathfrak{g},[]$,$) , denote the Lie bracket on \mathfrak{g}$ by the map $\mu: \wedge^{2} \mathfrak{g} \rightarrow \mathfrak{g}$. Note that $\mu$ is a 2 -cocycle in the Chevalley-Eilenberg of $\mathfrak{g}$ with coefficients in itself. A $(-\mu)$-twisted Rota-Baxter operator is called a Reynolds operator on the Lie algebra $\mathfrak{g}$.

2.13 Definition. Let $(\mathfrak{g},[]$,$) be a Lie algebra. A Reynolds operator on \mathfrak{g}$ is a linear map $R: \mathfrak{g} \rightarrow \mathfrak{g}$ satisfying

$$
[R x, R y]=R([R x, y]+[x, R y]-[R x, R y]), \quad \text { for } x, y \in \mathfrak{g} .
$$

It follows from Proposition 2.4 that a Reynolds operator $R: \mathfrak{g} \rightarrow \mathfrak{g}$ induces a new Lie algebra structure $\mathfrak{g}$ given by

$$
[x, y]_{R}:=[R x, y]+[x, R y]-[R x, R y], \text { for } x, y \in \mathfrak{g} .
$$

2.14 Example. Let $W$ be the Witt algebra generated by basis elements $\left\{l_{n}\right\}_{n \in \mathbb{Z}}$ and the Lie bracket given by

$$
\left[l_{m}, l_{n}\right]=(m-n) l_{m+n}, \text { for } m, n \geq 0 .
$$

Note that $W_{\geq 0}=\operatorname{span}\left\{l_{n} \mid n \geq 0\right\}$ is a Lie subalgebra of $W$. Then the linear map $R: W_{\geq 0} \rightarrow W_{\geq 0}$ defined by $R\left(l_{m}\right)=\frac{1}{m+1} l_{m}$, for $m \geq 0$, is a Reynolds operator on $W_{\geq 0}$. First observe that, for any $m, n \geq 0$,

$$
\left[R\left(l_{m}\right), R\left(l_{n}\right)\right]=\frac{1}{(m+1)(n+1)}\left[l_{m}, l_{n}\right]=\frac{(m-n)}{(m+1)(n+1)} l_{m+n} .
$$

On the other hand,

$$
\begin{aligned}
& R\left(\left[R\left(l_{m}\right), l_{n}\right]+\left[l_{m}, R\left(l_{n}\right)\right]-\left[R\left(l_{m}\right), R\left(l_{n}\right)\right]\right) \\
& =R\left(\frac{m-n}{m+1} l_{m+n}+\frac{m-n}{n+1} l_{m+n}-\frac{m-n}{(m+1)(n+1)} l_{m+n}\right) \\
& =\left(\frac{m-n}{m+1}+\frac{m-n}{n+1}-\frac{m-n}{(m+1)(n+1)}\right) \frac{1}{m+n+1} l_{m+n}=\frac{(m-n)}{(m+1)(n+1)} l_{m+n} .
\end{aligned}
$$

It follows from (8) and (9) that $R$ is a Reynolds operator on $W_{\geq 0}$. The induced Lie algebra structure on $W_{\geq 0}$ is given by

$$
\left[l_{m}, l_{n}\right]_{R}:=\left[R\left(l_{m}\right), l_{n}\right]+\left[l_{m}, R\left(l_{n}\right)\right]-\left[R\left(l_{m}\right), R\left(l_{n}\right)\right]=\frac{(m-n)(m+n+1)}{(m+1)(n+1)} l_{m+n} .
$$

Let $R: \mathfrak{g} \rightarrow \mathfrak{g}$ be a Reynolds operator on $\mathfrak{g}$ such that $R$ is invertible. Then it follows from (6) that

$$
R^{-1}[u, v]=\left[u, R^{-1} v\right]+\left[R^{-1} u, v\right]-[u, v], \quad \text { for } u, v \in \mathfrak{g}
$$

which implies that $\left(R^{-1}-\mathrm{id}\right)[u, v]=\left[\left(R^{-1}-\mathrm{id}\right)(u), v\right]+\left[u,\left(R^{-1}-\mathrm{id}\right)(v)\right]$, for $u, v \in \mathfrak{g}$. This shows that $\left(R^{-1}-\mathrm{id}\right): \mathfrak{g} \rightarrow \mathfrak{g}$ is a Lie algebra derivation. Conversely, if $d$ is a derivation such that (id $\left.+d\right): \mathfrak{g} \rightarrow \mathfrak{g}$ is invertible, then $(\mathrm{id}+d)^{-1}$ is a Reynolds operator. Even, if $(\mathrm{id}+d)$ is not invertible but the infinite sum $(\mathrm{id}+d)^{-1}=\sum_{n=0}^{\infty}(-1)^{n} d^{n}$ converges pointwise, then $(\mathrm{id}+d)^{-1}$ is a Reynolds operator. More precise statement is given below whose proof is similar to [27, Proposition 2.8].

2.15 Proposition. Let $\mathfrak{g}$ be a Lie algebra and $d: \mathfrak{g} \rightarrow \mathfrak{g}$ be a derivation. Suppose, for each $x \in \mathfrak{g}$, the infinite sum $\sum_{n=0}^{\infty}(-1)^{n} d^{n}(x)$ converges to a element in $\mathfrak{g}$. Then $R=\sum_{n=0}^{\infty}(-1)^{n} d^{n}$ is a Reynolds operator on $\mathfrak{g}$. 
It follows from the above proposition that if $d$ is a nilpotent derivation (more generally a locally nilpotent derivation) on $\mathfrak{g}$, then $R=\sum_{n=0}^{\infty}(-1)^{n} d^{n}$ is a Reynolds operator on $\mathfrak{g}$.

\section{Maurer-Cartan characterization of twisted Rota-Baxter operators and cohomology}

In this section, we construct an $L_{\infty}$-algebra whose Maurer-Cartan elements are $H$-twisted Rota-Baxter operators. This $L_{\infty}$-algebra consists of only bilinear and trilinear brackets, among which the bilinear bracket (which is constructed in [24] to characterize Rota-Baxter operators) itself satisfies the graded Jacobi identity. Such characterization of $H$-twisted Rota-Baxter operator $T$ allows us to introduce the cohomology of $T$. We also show that the cohomology of $T$ is equivalently described by the ChevalleyEilenberg cohomology of $M$ with coefficients in a suitable representation of $\mathfrak{g}$.

3.1 Definition. [16,17] An $L_{\infty}$-algebra is a graded vector space $L=\bigoplus_{i} L_{i}$ together with a collection $\left\{l_{k}: L^{\otimes k} \rightarrow L \mid \operatorname{deg}\left(l_{k}\right)=2-k\right\}_{k \geq 1}$ of multilinear maps satisfying the followings.

(i) Skew-symmetry: $l_{k}\left(x_{\sigma(1)}, \ldots, x_{\sigma(k)}\right)=(-1)^{\sigma} \epsilon(\sigma) l_{k}\left(x_{1}, \ldots, x_{k}\right)$, for $k \geq 1$ and $\sigma \in S_{k}$. Here $\epsilon(\sigma)=$ $\epsilon\left(\sigma ; x_{1}, \ldots, x_{k}\right)$ is the Koszul sign.

(ii) Higher Jacobi identities: for each $n \geq 1$, we have

$$
\sum_{i+j=n+1} \sum_{\sigma}(-1)^{\sigma} \epsilon(\sigma)(-1)^{i(j-1)} l_{j}\left(l_{i}\left(x_{\sigma(1)}, \ldots, x_{\sigma(i)}\right), x_{\sigma(i+1)}, \ldots, x_{\sigma(n)}\right)=0
$$

where $\sigma$ runs over all $(i, n-i)$-unshuffles with $i \geq 1$.

An element $\alpha \in L_{1}$ is said to be a Maurer-Cartan element (see for instance [21]) if it satisfies

$$
l_{1}(\alpha)+\frac{1}{2 !} l_{2}(\alpha, \alpha)-\frac{1}{3 !} l_{3}(\alpha, \alpha, \alpha)-\cdots=0 .
$$

Next we recall the graded Lie algebra constructed in [24]. Let $\mathfrak{g}$ be a Lie algebra and $M$ be a $\mathfrak{g}$-module. Denote the Lie bracket on $\mathfrak{g}$ by the map $\mu: \wedge^{2} \mathfrak{g} \rightarrow \mathfrak{g}$ and the $\mathfrak{g}$-module structure on $M$ by $\bullet: \mathfrak{g} \times M \rightarrow M$. Then the graded vector space $\bigoplus_{n \geq 0} \operatorname{Hom}\left(\wedge^{n} M, \mathfrak{g}\right)$ carries a graded Lie bracket given by

$$
\llbracket P, Q \rrbracket:=(-1)^{p}\left[[\mu+\bullet, P]_{\mathrm{NR}}, Q\right]_{\mathrm{NR}},
$$

for $P \in \operatorname{Hom}\left(\wedge^{p} M, \mathfrak{g}\right), Q \in \operatorname{Hom}\left(\wedge^{q} M, \mathfrak{g}\right)$, and $[,]_{\mathrm{NR}}$ is the Nijenhuis-Richardson bracket on skewsymmetric multilinear maps on the vector space $\mathfrak{g} \oplus M$. Explicitly,

$$
\begin{aligned}
\llbracket P, Q \rrbracket\left(u_{1}, \ldots, u_{p+q}\right)= & \sum_{\sigma \in S h(q, 1, p-1)}(-1)^{\sigma} P\left(Q\left(u_{\sigma(1)}, \ldots, u_{\sigma(q)}\right) \bullet u_{\sigma(q+1)}, u_{\sigma(q+2)}, \ldots, u_{\sigma(p+q)}\right) \\
& -(-1)^{p q} \sum_{\sigma \in S h(p, 1, q-1)}(-1)^{\sigma} Q\left(P\left(u_{\sigma(1)}, \ldots, u_{\sigma(p)}\right) \bullet u_{\sigma(p+1)}, u_{\sigma(p+2)}, \ldots, u_{\sigma(p+q)}\right) \\
& +(-1)^{p q} \sum_{S h(p, q)}(-1)^{\sigma}\left[P\left(u_{\sigma(1)}, \ldots, u_{\sigma(p)}\right), Q\left(u_{\sigma(n+1)}, \ldots, u_{\sigma(p+q)}\right)\right] .
\end{aligned}
$$

Hence, for any $T \in \operatorname{Hom}(M, \mathfrak{g})$,

$$
\llbracket T, T \rrbracket(u, v)=2\{T(T(u) \bullet v-T(v) \bullet u)-[T u, T v]\}, \text { for } u, v \in M
$$

This shows that Maurer-Cartan elements on this graded Lie algebra are Rota-Baxter operators [24].

Let $H$ be a 2-cocycle in the cohomology of $\mathfrak{g}$ with coefficients in $M$. Following the case of twisted Poisson manifolds [7], here we introduce a ternary degree -1 bracket $\llbracket, ~ \rrbracket$ on the graded space $\bigoplus_{n \geq 0} \operatorname{Hom}\left(\wedge^{n} M, \mathfrak{g}\right)$ 
as follows. For $P \in \operatorname{Hom}\left(\wedge^{p} M, \mathfrak{g}\right), Q \in \operatorname{Hom}\left(\wedge^{q} M, \mathfrak{g}\right)$, and $R \in \operatorname{Hom}\left(\wedge^{r} M, \mathfrak{g}\right)$, we define $\llbracket P, Q, R \rrbracket \in$ $\operatorname{Hom}\left(\wedge^{p+q+r-1} M, \mathfrak{g}\right)$ by

$$
\begin{aligned}
& \llbracket P, Q, R \rrbracket\left(u_{1}, \ldots, u_{p+q+r-1}\right) \\
& =(-1)^{p q r} \frac{1}{2}\left\{\sum_{\sigma \in S h(q, r, p-1)}(-1)^{\sigma} P\left(H\left(Q\left(u_{\sigma(1)}, \ldots, u_{\sigma(q)}\right), R\left(u_{\sigma(q+1)}, \ldots, u_{\sigma(q+r)}\right)\right), u_{\sigma(q+r+1)} \ldots, u_{\sigma(p+q+r-1)}\right)\right. \\
& -(-1)^{q r} \sum_{\sigma \in S h(r, q, p-1)}(-1)^{\sigma} P\left(H\left(R\left(u_{\sigma(1)}, \ldots, u_{\sigma(r)}\right), Q\left(u_{\sigma(r+1)}, \ldots, u_{\sigma(q+r)}\right)\right), u_{\sigma(q+r+1)} \ldots, u_{\sigma(p+q+r-1)}\right) \\
& -(-1)^{p q} \sum_{\sigma \in S h(p, r, q-1)}(-1)^{\sigma} Q\left(H\left(P\left(u_{\sigma(1)}, \ldots, u_{\sigma(p)}\right), R\left(u_{\sigma(p+1)}, \ldots, u_{\sigma(p+r)}\right)\right), u_{\sigma(p+r+1)} \ldots, u_{\sigma(p+q+r-1)}\right) \\
& +(-1)^{p(q+r)} \sum_{\sigma \in S h(r, p, q-1)}(-1)^{\sigma} Q\left(H\left(R\left(u_{\sigma(1)}, \ldots, u_{\sigma(r)}\right), P\left(u_{\sigma(r+1)}, \ldots, u_{\sigma(p+r)}\right)\right), u_{\sigma(p+r+1)} \ldots, u_{\sigma(p+q+r-1)}\right) \\
& +(-1)^{(p+q) r} \sum_{\sigma \in S h(p, q, r-1)}(-1)^{\sigma} R\left(H\left(P\left(u_{\sigma(1)}, \ldots, u_{\sigma(p)}\right), Q\left(u_{\sigma(p+1)}, \ldots, u_{\sigma(p+q)}\right)\right), u_{\sigma(p+q+1)} \ldots, u_{\sigma(p+q+r-1)}\right) \\
& \left.-(-1)^{p q+q r+r p} \sum_{\sigma \in S h(q, p, r-1)}(-1)^{\sigma} R\left(H\left(Q\left(u_{\sigma(1)}, \ldots, u_{\sigma(q)}\right), P\left(u_{\sigma(q+1)}, \ldots, u_{\sigma(p+q)}\right)\right), u_{\sigma(p+q+1)} \ldots, u_{\sigma(p+q+r-1)}\right)\right\} .
\end{aligned}
$$

The bracket $\llbracket P, Q, R \rrbracket$ is obviously graded skew-symmetric. Moreover, the binary bracket $\llbracket$, $\rrbracket$ and the ternary bracket $\llbracket,, \rrbracket$ are compatible in the sense of $L_{\infty}$-algebra. This follows as $H$ is a 2-cocycle. In summary, we obtain the following.

3.2 Theorem. Let $\mathfrak{g}$ be a Lie algebra, $M$ be a $\mathfrak{g}$-module and $H$ be a 2-cocycle in the cohomology of $\mathfrak{g}$ with coefficients in $M$. Then the graded vector space $\bigoplus_{n \geq 0} \operatorname{Hom}\left(\wedge^{n} M, \mathfrak{g}\right)$ is an $L_{\infty}$-algebra with

$$
l_{1}=0, \quad l_{2}=\llbracket, \rrbracket, \quad l_{3}=\llbracket, \rrbracket,
$$

and higher maps are trivial. A linear map $T: M \rightarrow \mathfrak{g}$ is a $H$-twisted Rota-Baxter operator if and only if $T \in \operatorname{Hom}(M, \mathfrak{g})$ is a Maurer-Cartan element in the above $L_{\infty}$-algebra $\left(\bigoplus_{n \geq 0} \operatorname{Hom}\left(\wedge^{n} M, \mathfrak{g}\right), \llbracket \mathbb{}, \mathbb{\rrbracket}, \llbracket,, \mathbb{\rrbracket}\right)$.

Proof. The first part follows from the previous discussions. For the second part, we observe that for any $T \in \operatorname{Hom}(M, \mathfrak{g})$,

$$
\llbracket T, T, T \rrbracket(u, v)=-6 T(H(T u, T v)) .
$$

Hence from (11) and (13), we get

$$
\left(\frac{1}{2} \llbracket T, T \rrbracket-\frac{1}{6} \llbracket T, T, T \rrbracket\right)(u, v)=T(T(u) \bullet v-T(v) \bullet u)-[T u, T v]+T(H(T u, T v)) .
$$

This shows that $T$ is a $H$-twisted Rota-Baxter operator if and only if $T$ is a Maurer-Cartan element in the $L_{\infty}$-algebra.

The above characterization of a $H$-twisted Rota-Baxter operator $T$ allows us to define a cohomology associated to $T$. More precisely, we define $C_{T}^{n}(M, \mathfrak{g})=\operatorname{Hom}\left(\wedge^{n} M, \mathfrak{g}\right)$, for $n \geq 0$, and the differential $d_{T}: C_{T}^{n}(M, \mathfrak{g}) \rightarrow C_{T}^{n+1}(M, \mathfrak{g})$ by

$$
d_{T}(f)=\llbracket T, f \rrbracket-\frac{1}{2} \llbracket T, T, f \rrbracket, \text { for } f \in C_{T}^{n}(M, \mathfrak{g}) .
$$

The corresponding cohomology groups

$$
H_{T}^{n}(M, \mathfrak{g})=\frac{Z_{T}^{n}(M, \mathfrak{g})}{B_{T}^{n}(M, \mathfrak{g})}=\frac{\left\{f \in C_{T}^{n}(M, \mathfrak{g}) \mid d_{T} f=0\right\}}{\left\{d_{T} g \mid g \in C_{T}^{n-1}(M, \mathfrak{g})\right\}}, \text { for } n \geq 0
$$


are called the cohomology of the $H$-twisted Rota-Baxter operator $T$.

Given an $L_{\infty}$-algebra and a Maurer-Cartan element, Getzler [9] construct a new $L_{\infty}$-algebra twisted by the Maurer-Cartan element. Here we will follow the conventions of [21]. This rephrases in our context as follows.

3.3 Proposition. Let $T$ be a $H$-twisted Rota-Baxter operator. Then $\bigoplus_{n \geq 0} \operatorname{Hom}\left(\wedge^{n} M, \mathfrak{g}\right)$ carries an $L_{\infty^{-}}$ algebra with structure maps

$$
l_{1}(P)=d_{T}(P), \quad \llbracket P, Q \rrbracket_{T}=\llbracket P, Q \rrbracket-\llbracket T, P, Q \rrbracket, \quad \llbracket P, Q, R \rrbracket_{T}=\llbracket P, Q, R \rrbracket
$$

and trivial higher brackets. We call this as the twisted $L_{\infty}$-algebra twisted by $T$.

3.4 Theorem. Let $T$ be a $H$-twisted Rota-Baxter operator. For any linear map $T^{\prime}: M \rightarrow \mathfrak{g}$, the sum $T+T^{\prime}$ is a $H$-twisted Rota-Baxter operator if and only if $T^{\prime}$ is a Maurer-Cartan element in the above twisted $L_{\infty}$-algebra .

Proof. We have

$$
\begin{aligned}
& \frac{1}{2} \llbracket T+T^{\prime}, T+T^{\prime} \rrbracket-\frac{1}{6} \llbracket T+T^{\prime}, T+T^{\prime}, T+T^{\prime} \rrbracket \\
& =\frac{1}{2} \llbracket T, T^{\prime} \rrbracket+\frac{1}{2} \llbracket T^{\prime}, T \rrbracket+\frac{1}{2} \llbracket T^{\prime}, T^{\prime} \rrbracket-\frac{1}{6} \llbracket T, T, T^{\prime} \rrbracket-\frac{1}{6} \llbracket T, T^{\prime}, T \rrbracket-\frac{1}{6} \llbracket T^{\prime}, T, T \rrbracket \\
& -\frac{1}{6} \llbracket T, T^{\prime}, T^{\prime} \rrbracket-\frac{1}{6} \llbracket T^{\prime}, T, T^{\prime} \rrbracket-\frac{1}{6} \llbracket T^{\prime}, T^{\prime}, T \rrbracket-\frac{1}{6} \llbracket T^{\prime}, T^{\prime}, T^{\prime} \rrbracket \\
& =\left(\llbracket T, T^{\prime} \rrbracket-\frac{1}{2} \llbracket T, T, T^{\prime} \rrbracket\right)+\frac{1}{2}\left(\llbracket T^{\prime}, T^{\prime} \rrbracket-\llbracket T, T^{\prime}, T^{\prime} \rrbracket\right)-\frac{1}{6} \llbracket T^{\prime}, T^{\prime}, T^{\prime} \rrbracket \\
& =d_{T}\left(T^{\prime}\right)+\frac{1}{2} \llbracket T^{\prime}, T^{\prime} \rrbracket_{T}-\frac{1}{6} \llbracket T^{\prime}, T^{\prime}, T^{\prime} \rrbracket_{T} .
\end{aligned}
$$

This proves the statement.

\subsection{Another interpretation of the cohomology}

Let $T: M \rightarrow \mathfrak{g}$ be a $H$-twisted Rota-Baxter operator. We have seen in Proposition 2.4 that the vector space $M$ carries a Lie algebra structure with bracket

$$
[u, v]_{T}:=T(u) \bullet v-T(v) \bullet u+H(T u, T v), \text { for } u, v \in M .
$$

In this subsection, we show that the cohomology of $T$ can be interpreted as the Chevalley-Eilenberg cohomology of $\left(M,[,]_{T}\right)$ with coefficients in a suitable module structure on $\mathfrak{g}$.

We start with the following.

3.5 Proposition. The linear map $\boldsymbol{\boldsymbol { \bullet }}: M \times \mathfrak{g} \rightarrow \mathfrak{g}$ defined by

$$
u \overline{\boldsymbol{\bullet}} x=[T u, x]+T(x \bullet u+H(x, T u)), \text { for } u \in M, x \in \mathfrak{g}
$$

is a representation of the Lie algebra $\left(M,[,]_{T}\right)$ on the vector space $\mathfrak{g}$. 
Proof. By a direct calculation using the definition of $\mathbf{-}$, we get for $u, v \in M, x \in \mathfrak{g}$,

$$
\begin{aligned}
u & \bar{\bullet}(v \bar{\bullet} x)-v \boldsymbol{\bullet}(u \bar{\bullet} x)-[u, v]_{T} \mathbf{\bullet} x \\
= & {[T u,[T v, x]+T([T v, x] \bullet u)+T H([T v, x], T u)+[T u, T(x \bullet v)]+T(T(x \bullet v) \bullet u)} \\
& +T H(T(x \bullet v), T u)+[T u, T H(x, T v)]+T(T H(x, T v) \bullet u)+T H(T H(x, T v), T u)-[T v,[T u, x]] \\
& -T([T u, x] \bullet v)-T H([T u, x], T v)-[T v, T(x \bullet u)]-T(T(x \bullet u) \bullet v)-T H(T(x \bullet u), T v) \\
& -[T v, T H(x, T u)]-T(T H(x, T u) \bullet v)-T H(T H(x, T u), T v)-[[T u, T v], x]-T(x \bullet(T u \bullet v)) \\
& +T(x \bullet(T v \bullet u))-T(x \bullet H(T u, T v))-T H(x,[T u, T v]) \\
= & T([T v, x] \bullet u)+T H([T v, x], T u)+T((T u) \bullet(x \bullet v))+T H(T u, T(x \bullet v))+T H(T(x \bullet v), T u) \\
& +T(T u \bullet H(x, T v))+T H(T u, T H(x, T v))+T H(T H(x, T v), T u)-T([T u, x] \bullet v)-T H([T u, x], T v) \\
& -T((T v) \bullet(x \bullet u))-T H(T v, T(x \bullet u))-T H(T(x \bullet u), T v)-T(T v \bullet H(x, T u))-T H(T v, T H(x, T u)) \\
& -T H(T H(x, T u), T v)-T(x \bullet(T u \bullet v))+T(x \bullet(T v \bullet u))-T(x \bullet H(T u, T v))-T H(x,[T u, T v]) \\
& \text { after cancellations } T H([T v, x], T u)+T(T u \bullet H(x, T v))-T H([T u, x], T v)-T(T v \bullet H(x, T u)) \\
& =T(x \bullet H(T u, T v))-T H(x,[T u, T v])) \\
= & -T\left(\left(\delta_{\mathrm{CE}} H\right)(T u, T v, x)\right)=0 .
\end{aligned}
$$

Hence the result follows.

It follows from the above proposition that we may consider the Chevalley-Eilenberg cohomology of the Lie algebra $\left(M,[,]_{T}\right)$ with coefficients in the representation $\mathfrak{g}$. More precisely, we define

$$
C_{\mathrm{CE}}^{n}(M, \mathfrak{g}):=\operatorname{Hom}\left(\wedge^{n} M, \mathfrak{g}\right), \text { for } n \geq 0
$$

and the differential $\delta_{\mathrm{CE}}: C_{\mathrm{CE}}^{n}(M, \mathfrak{g}) \rightarrow C_{\mathrm{CE}}^{n+1}(M, \mathfrak{g})$ by

$$
\begin{aligned}
& \left(\delta_{\mathrm{CE}} f\right)\left(u_{1}, \ldots, u_{n+1}\right) \\
& =\sum_{i=1}^{n+1}(-1)^{i+1}\left[T u_{i}, f\left(u_{1}, \ldots, \widehat{u_{i}}, \ldots, u_{n+1}\right)\right]+\sum_{i=1}^{n+1}(-1)^{i+1} T\left(f\left(u_{1}, \ldots, \widehat{u_{i}}, \ldots, u_{n+1}\right) \bullet u_{i}\right) \\
& +\sum_{i=1}^{n+1}(-1)^{i+1} T H\left(f\left(u_{1}, \ldots, \widehat{u_{i}}, \ldots, u_{n+1}\right), T u_{i}\right) \\
& +\sum_{i \leq i<j \leq n+1}(-1)^{i+j} f\left(T\left(u_{i}\right) \bullet u_{j}-T\left(u_{j}\right) \bullet u_{i}+H\left(T u_{i}, T u_{j}\right), u_{1}, \ldots, \widehat{u_{i}}, \ldots, \widehat{u_{j}}, \ldots, u_{n+1}\right),
\end{aligned}
$$

for $f \in C_{\mathrm{CE}}^{n}(M, \mathfrak{g})$ and $u_{1}, \ldots, u_{n+1} \in M$.

3.6 Proposition. Let $T: M \rightarrow \mathfrak{g}$ be a H-twisted Rota-Baxter operator. Then for any $f \in \operatorname{Hom}\left(\wedge^{n} M, \mathfrak{g}\right)$,

$$
d_{T} f=(-1)^{n} \delta_{\mathrm{CE}} f .
$$

Consequently, the cohomology of $T$ is isomorphic to the Chevalley-Eilenberg cohomology of $\left(M,[,]_{*}\right)$ with coefficients in $\mathfrak{g}$. 
Proof. In [24, Proposition 3.3] the authors showed that

$$
\begin{aligned}
& \llbracket T, f \rrbracket\left(u_{1}, \ldots, u_{n+1}\right) \\
& =(-1)^{n}\left\{\sum_{i=1}^{n+1}(-1)^{i+1}\left[T u_{i}, f\left(u_{1}, \ldots, \widehat{u_{i}}, \ldots, u_{n+1}\right)\right]+\sum_{i=1}^{n+1}(-1)^{i+1} T\left(f\left(u_{1}, \ldots, \widehat{u_{i}}, \ldots, u_{n+1}\right) \bullet u_{i}\right)\right. \\
& \left.\quad+\sum_{1 \leq i<j \leq n+1}(-1)^{i+j} f\left(T\left(u_{i}\right) \bullet u_{j}-T\left(u_{j}\right) \bullet u_{i}, u_{1}, \ldots, \widehat{u_{i}}, \ldots, \widehat{u_{j}}, \ldots, u_{n+1}\right)\right\} .
\end{aligned}
$$

Here we observe that

$$
\begin{array}{rl}
\llbracket T, T, f \rrbracket\left(u_{1}, \ldots, u_{n+1}\right)=(-1)^{n} \frac{1}{2}\{ & 2 \sum_{i=1}^{n+1}(-1)^{i+1} T\left(H\left(T u_{i}, f\left(u_{1}, \ldots, \widehat{u_{i}}, \ldots, u_{n+1}\right)\right)\right) \\
& -(-1)^{n} 2 \sum_{i=1}^{n+1}(-1)^{n-i+1} T\left(H\left(f\left(u_{1}, \ldots, \widehat{u_{i}}, \ldots, u_{n+1}\right), T u_{i}\right)\right) \\
& +2 \sum_{1 \leq i<j \leq n+1}(-1)^{i+j-1} f\left(H\left(T u_{i}, T u_{j}\right), u_{1}, \ldots, \widehat{u_{i}}, \ldots, \widehat{u_{j}}, \ldots, u_{n+1}\right) \\
& \left.+2 \sum_{1 \leq i<j \leq n+1}(-1)^{i+j} f\left(H\left(T u_{j}, T u_{i}\right), u_{1}, \ldots, \widehat{u_{i}}, \ldots, \widehat{u_{j}}, \ldots, u_{n+1}\right)\right\} \\
=-(-1)^{n} & 2\left\{\sum_{i=1}^{n+1}(-1)^{i+1} T H\left(f\left(u_{1}, \ldots, \widehat{u_{i}}, \ldots, u_{n+1}\right), T u_{i}\right)\right. \\
& \left.+\sum_{i \leq i<j \leq n+1}(-1)^{i+j} f\left(H\left(T u_{i}, T u_{j}\right), u_{1}, \ldots, \widehat{u_{i}}, \ldots, \widehat{u_{j}}, \ldots, u_{n+1}\right)\right\} .
\end{array}
$$

Hence $d_{T} f=\llbracket T, f \rrbracket-\frac{1}{2} \llbracket T, T, f \rrbracket=(-1)^{n} \delta_{\mathrm{CE}} f$. This proves the result.

\section{Deformations of twisted Rota-Baxter operators}

In this section, we study linear and formal deformations of a $H$-twisted Rota-Baxter operator. We introduce Nijenhuis elements associated with a $H$-twisted Rota-Baxter operator that arise from trivial deformations. Finally, we consider rigidity of a $H$-twisted Rota-Baxter operator and provide a sufficient condition for the same in terms of Nijenhuis elements.

\subsection{Linear deformations}

Let $\mathfrak{g}$ be a Lie algebra, $M$ be a $\mathfrak{g}$-module and $H: \wedge^{2} \mathfrak{g} \rightarrow M$ be a 2-cocycle in the Chevalley-Eilenberg cohomology of $\mathfrak{g}$ with coefficients in $M$. Suppose $T: M \rightarrow \mathfrak{g}$ is a $H$-twisted Rota-Baxter operator.

4.1 Definition. A linear deformation of $T$ consists of a parametrized sum $T_{t}=T+t T_{1}$, for some $T_{1} \in$ $\operatorname{Hom}(M, \mathfrak{g})$ such that $T_{t}$ is a $H$-twisted Rota-Baxter operator for all values of $t$. In this case, we say that $T_{1}$ generates a linear deformation of $T$.

Thus, in a linear deformation $T_{t}=T+t T_{1}$, we must have the followings

$$
\begin{aligned}
{\left[T(u), T_{1}(v)\right]+\left[T_{1}(u), T(v)\right]=} & T_{1}(T(u) \bullet v-T(v) \bullet u+H(T u, T v)) \\
& +T\left(T_{1}(u) \bullet v-T_{1}(v) \bullet u+H\left(T_{1}(u), T v\right)+H\left(T u, T_{1}(v)\right)\right) \\
{\left[T_{1}(u), T_{1}(v)\right]=} & T\left(H\left(T_{1}(u), T_{1}(v)\right)\right)+T_{1}\left(T_{1}(u) \bullet v-T_{1}(v) \bullet u+H\left(T_{1}(u), T v\right)+H\left(T u, T_{1}(v)\right)\right) \\
0= & T_{1}\left(H\left(T_{1}(u), T_{1}(v)\right)\right) .
\end{aligned}
$$


Note that the identity (14) implies that $T_{1}$ is a 1-cocycle in the cohomology of $T$. Hence, $T_{1}$ defines a cohomology class in $H_{T}^{1}(M, \mathfrak{g})$.

4.2 Definition. Two linear deformations $T_{t}=T+t T_{1}$ and $T_{t}^{\prime}=T+t T_{1}^{\prime}$ of a $H$-twisted Rota-Baxter operator $T$ are said to be equivalent if there exists an element $x \in \mathfrak{g}$ such that the pair

$$
\left(\phi_{t}=\operatorname{id}_{\mathfrak{g}}+t[x,-], \psi_{t}=\operatorname{id}_{M}+t(x \bullet-+H(x, T-))\right)
$$

defines a morphism of $H$-twisted Rota-Baxter operators from $T_{t}$ to $T_{t}^{\prime}$.

Thus, it follows that $\phi_{t}$ is a Lie algebra homomorphism which implies that

$$
[[x, y],[x, z]]=0, \quad \text { for } y, z \in \mathfrak{g} \text {. }
$$

The condition $\psi_{t}(y \bullet u)=\phi_{t}(y) \bullet \psi_{t}(u)$ implies that

$$
\left\{\begin{array}{l}
H(x, T(y \bullet u))=y \bullet H(x, T u), \\
{[x, y] \bullet(x \bullet u+H(x, T u))=0 .}
\end{array}\right.
$$

Finally, the conditions $\psi_{t} \circ H=H \circ\left(\phi_{t} \otimes \phi_{t}\right)$ and $\phi_{t} \circ T_{t}=T_{t}^{\prime} \circ \psi_{t}$ are respectively equivalent to

$$
\begin{gathered}
\left\{\begin{array}{l}
x \bullet H(y, z)+H(x, T H(y, z))=H([x, y], z)+H(y,[x, z]), \\
H([x, y],[x, z])=0
\end{array}\right. \\
\qquad\left\{\begin{array}{l}
T_{1}(u)+[x, T u]=T(x \bullet u+H(x, T u))+T_{1}^{\prime}(u), \\
{\left[x, T_{1}(u)\right]=T_{1}^{\prime}(x \bullet u+H(x, T u)) .}
\end{array}\right.
\end{gathered}
$$

Note that the above identities hold for all $y, x \in \mathfrak{g}$ and $u \in M$. It is easy to see from the first condition of (20) that $T_{1}(u)-T_{1}^{\prime}(u)=d_{T}(x)(u)$. Therefore, we get the following.

4.3 Theorem. Let $T_{t}=T+t T_{1}$ and $T_{t}=T+t T_{1}^{\prime}$ be two equivalent linear deformations of a $H$-twisted Rota-Baxter operator. Then $T_{1}$ and $T_{1}^{\prime}$ defines the same cohomology class in $H_{T}^{1}(M, \mathfrak{g})$.

4.4 Definition. A linear deformation $T_{t}=T+t T_{1}$ of a $H$-twisted Rota-Baxter operator is said to be trivial if $T_{t}$ is equivalent to the undeformed deformation $T_{t}^{\prime}=T$.

We will now define Nijenhuis elements associated with a $H$-twisted Rota-Baxter operator.

4.5 Definition. Let $T$ be a $H$-twisted Rota-Baxter operator. An element $x \in \mathfrak{g}$ is called a Nijenhuis element associated to $T$ if $x$ satisfies

$$
[x, u \bar{\bullet} x]=0
$$

and (17), (18), (19) holds.

We denote by $\operatorname{Nij}(T)$ the set of all Nijenhuis elements associated with the $H$-twisted Rota-Baxter operator. It follows from the above definition that a trivial linear deformation gives rise to a Nijenhuis element which is the motivation to introduce the definition. In the next subsection, we obtain a sufficient condition for the rigidity of a twisted Rota-Baxter operator in terms of Nijenhuis elements.

\subsection{Formal deformations}

In this subsection, we consider formal deformations of $H$-twisted Rota-Baxter operators generalizing the classical deformation theory of Gerstenhaber [8]. 
Let $\mathfrak{g}$ be a Lie algebra, $M$ be a $\mathfrak{g}$-module and $H$ be a 2-cocycle in the Chevalley-Eilenberg cohomology of $\mathfrak{g}$ with coefficients in $M$. Let $T: M \rightarrow \mathfrak{g}$ be a $H$-twisted Rota-Baxter operator.

Consider the space $\mathfrak{g}[[t]]$ of formal power series in $t$ with coefficients from $\mathfrak{g}$. Then $\mathfrak{g}[[t]]$ is a $\mathbb{K}[[t]]$ module. Note that the Lie algebra structure on $\mathfrak{g}$ induces a Lie algebra structure on $\mathfrak{g}[[t]]$ by $\mathbb{K}[[t]]$ bilinearity. Moreover, the $\mathfrak{g}$-module structure on $M$ induces a $\mathfrak{g}[[t]]$-module structure on $M[[t]]$. Similarly, the 2-cocycle $H$ can be extended to a 2-cocycle (denoted by the same notation $H$ ) on the Lie algebra $\mathfrak{g}[[t]]$ with coefficients in $M[[t]]$.

4.6 Definition. A formal deformation of $T$ consists of a formal sum $T_{t}=\sum_{i=0}^{\infty} t^{i} T_{i}$ (with $T_{i} \in \operatorname{Hom}(M, \mathfrak{g})$ for each $i$ and $T_{0}=T$ ) such that $T_{t}$ is a $H$-twisted Rota-Baxter operator.

Thus, in a formal deformation as above, the following system of equations must hold: for $n \geq 0$,

$$
\sum_{i+j=n}\left[T_{i}(u), T_{j}(v)\right]=\sum_{i+j=n} T_{i}\left(T_{j}(u) \bullet v-T_{j}(v) \bullet u\right)+\sum_{i+j+k=n} T_{i}\left(H\left(T_{j}(u), T_{k}(v)\right)\right) .
$$

These equations are called deformation equations. Note that (21) holds for $n=0$ as $T_{0}=T$ is a $H$-twisted Rota-Baxter operator. For $n=1$, we get

$$
\begin{aligned}
{\left[T(u), T_{1}(v)\right]+\left[T_{1}(u), T(v)\right]=} & T_{1}(T(u) \bullet v-T(v) \bullet u+H(T u, T v)) \\
& +T\left(T_{1}(u) \bullet v-T_{1}(v) \bullet u+H\left(T_{1}(u), T v\right)+H\left(T u, T_{1}(v)\right)\right) .
\end{aligned}
$$

This implies that $\left(d_{T}\left(T_{1}\right)\right)(u, v)=0$. Hence the linear term $T_{1}$ is a 1-cocycle in the cohomology of $T$, called the infinitesimal of the deformation $T_{t}$.

In the following, we define an equivalence between two formal deformations generalizing the equivalence between linear deformations.

4.7 Definition. Let $T_{t}=\sum_{i=0}^{\infty} t^{i} T_{i}$ and $T_{t}^{\prime}=\sum_{i=0}^{\infty} t^{i} T_{i}^{\prime}$ be two formal deformations of a $H$-twisted RotaBaxter operator. They are said to be equivalent if there exists an element $x \in \mathfrak{g}$, linear maps $\phi_{i} \in \operatorname{Hom}(\mathfrak{g}, \mathfrak{g})$ and $\psi \in \operatorname{Hom}(M, M)$ for $i \geq 2$ such that the pair

$$
\left(\phi_{t}=\mathrm{id}_{\mathfrak{g}}+t[x,-]+\sum_{i=2}^{\infty} t^{i} \phi_{i}, \psi_{t}=\operatorname{id}_{M}+t(x \bullet-+H(x, T-))+\sum_{i=2}^{\infty} t^{i} \psi_{i}\right)
$$

is a morphism of $H$-twisted Rota-Baxter operators from $T_{t}$ to $T_{t}^{\prime}$.

Thus, it follows that $\left(\phi_{t}, \psi_{t}\right)$ satisfies the conditions of Definition 2.5. In particular, the condition $\left(\phi_{t} \circ T_{t}\right)(u)=\left(T_{t}^{\prime} \circ \psi_{t}\right)(u)$ implies that (by equating coefficients of $\left.t\right)$

$$
T_{1}(u)-T_{1}^{\prime}(u)=[T u, x]+T(x \bullet u+H(x, T u))=\left(d_{T}(x)\right)(u) .
$$

Hence, we get the following.

4.8 Theorem. If $T_{t}=\sum_{i=0}^{\infty} t^{i} T_{i}$ and $T_{t}^{\prime}=\sum_{i=0}^{\infty} t^{i} T_{i}^{\prime}$ are two equivalent formal deformations of a $H$ twisted Rota-Baxter operator $T$, then $T_{1}$ and $T_{1}^{\prime}$ defines the same cohomology class in $H_{T}^{1}(M, \mathfrak{g})$.

4.9 Definition. A $H$-twisted Rota-Baxter operator $T$ is said to be rigid if any formal deformation of $T$ is equivalent to the undeformed one $T_{t}^{\prime}=T$.

The next result gives a sufficient condition for the rigidity of a $H$-twisted Rota-Baxter operator.

4.10 Theorem. Let $T$ be a $H$-twisted Rota-Baxter operator. If $Z_{T}^{1}(M, \mathfrak{g})=d_{T}(\operatorname{Nij}(T))$ then $T$ is rigid.

Proof. Let $T_{t}=\sum_{i=0}^{\infty} t^{i} T_{i}$ be any formal deformation of $T$. Then we have seen that $T_{1}$ is a 1-cocycle in the cohomology of $T$, i.e. $T_{1} \in Z_{T}^{1}(M, \mathfrak{g})$. Thus, from the hypothesis, we get a Nijenhuis element $x \in \operatorname{Nij}(T)$ 
such that $T_{1}=d_{T}(x)$. We take

$$
\phi_{t}=\mathrm{id}_{\mathfrak{g}}+t[x,-], \quad \psi_{t}=\mathrm{id}_{M}+t(x \bullet-+H(x, T-)),
$$

and define $T_{t}^{\prime}=\phi_{t} \circ T_{t} \circ \psi_{t}^{-1}$. Then $T_{t}^{\prime}$ is a deformation equivalent to $T_{t}$. We claim that the coefficient of $t$ in $T_{t}^{\prime}$ is trivial. To see this, we observe that

$$
\begin{aligned}
T_{t}^{\prime}(u) \quad\left(\bmod t^{2}\right) & =\left(\operatorname{id}_{\mathfrak{g}}+t[x,-]\right) \circ\left(T+t T_{1}\right) \circ\left(\operatorname{id}_{M}-t(x \bullet-+H(x, T-))(u) \quad\left(\bmod t^{2}\right)\right. \\
& =\left(\operatorname{id}_{\mathfrak{g}}+t[x,-]\right)\left(T u+t T_{1}(u)-t T(x \bullet u+H(x, T u))\right) \quad\left(\bmod t^{2}\right) \\
& =T u+t\left(T_{1}(u)-T(x \bullet u)-T H(x, T u)+[x, T u]\right) \\
& =T u \quad\left(\operatorname{as} T_{1}(u)=d_{T}(x)(u)\right) .
\end{aligned}
$$

This proves the claim. By applying the same process repeatedly, we get that $T_{t}$ is equivalent to $T$. Hence $T$ is rigid.

\section{Applications}

\subsection{Reynolds operators}

As Reynolds operators on a Lie algebra $\mathfrak{g}$ are a particular case of $H$-twisted Rota-Baxter operators, we can apply the results of previous sections to Reynolds operators.

The following result is the Reynolds operator analogue of Theorem 3.2.

5.1 Theorem. Let $(\mathfrak{g}, \mu=[]$,$) be a Lie algebra. Then the graded vector space \bigoplus_{n} \operatorname{Hom}\left(\wedge^{n} \mathfrak{g}, \mathfrak{g}\right)$ carries an $L_{\infty}$-algebra structure with only nontrivial $l_{2}$ and $l_{3}$ that are given by (10) and (12) with $H=-\mu$. Moreover, a linear map $R: \mathfrak{g} \rightarrow \mathfrak{g}$ is a Reynolds operator on $\mathfrak{g}$ if and only if $R$ is a Maurer-Cartan element in the $L_{\infty}$-algebra.

Given a Reynolds operator $R$ on the Lie algebra $\mathfrak{g}$, the cohomology induced from the corresponding Maurer-Cartan element is called the cohomology of $R$. This cohomology of $R$ can also be seen as the Chevalley-Eilenberg cohomology of a certain Lie algebra with coefficients in a representation. More precisely, if $R: \mathfrak{g} \rightarrow \mathfrak{g}$ is a Reynolds operator, then $\mathfrak{g}$ has a new Lie algebra structure with bracket $[,]_{R}$ given by $(7)$. The Lie algebra $\left(\mathfrak{g},[,]_{R}\right)$ has a representation on $\mathfrak{g}$ itself by the following

$$
x \overline{\boldsymbol{\bullet}}_{R} y:=[R x, y]+R([y, x]-[y, R x]), \quad \text { for } x, y \in \mathfrak{g} .
$$

Then the corresponding Chevalley-Eilenberg cohomology is isomorphic to the cohomology of $R$.

Deformations of a Reynolds operator can also be defined as of Section 4. Such deformations are governed by the cohomology of the Reynolds operator.

\subsection{Twisted triangular r-matrices}

In this subsection, we introduce cohomology of twisted triangular $r$-matrices from the points of view of twisted Rota-Baxter operators. Let $\mathfrak{g}$ be a Lie algebra. Consider the coadjoint representation $\mathfrak{g}^{*}$ of the Lie algebra $\mathfrak{g}$. By considering the coadjoint representation in Theorem 3.2, we get the following.

5.2 Theorem. Let $\mathfrak{g}$ be a Lie algebra and $\psi \in \wedge^{3} \mathfrak{g}^{*}$ be a 3 -cocycle of $\mathfrak{g}$ with coefficients in $\mathbb{K}$. Then the graded vector space $\bigoplus_{n \geq 0} \operatorname{Hom}\left(\wedge^{n} \mathfrak{g}^{*}, \mathfrak{g}\right)$ carries an $L_{\infty}$-algebra structure given by

$$
l_{1}=0, \quad l_{2}=\llbracket, \rrbracket, \quad l_{3}=\llbracket, \rrbracket
$$




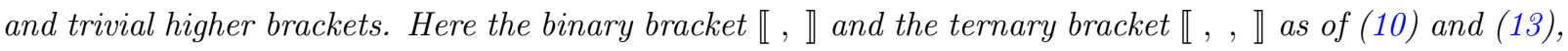
and we use $H=\psi^{\sharp}: \wedge^{2} \mathfrak{g} \rightarrow \mathfrak{g}^{*}$ which is a 2-cocycle of $\mathfrak{g}$ with coefficients in the coadjoint representation $\mathfrak{g}^{*}$. An element $\mathbf{r} \in \wedge^{2} \mathfrak{g}$ is a $\psi$-twisted triangular r-matrix if and only if $\mathbf{r}^{\sharp}: \mathfrak{g}^{*} \rightarrow \mathfrak{g}$ is a Maurer-Cartan element in the above $L_{\infty}$-algebra.

It follows from the above theorem that a $\psi$-twisted triangular $r$-matrix $\mathbf{r} \in \wedge^{2} \mathfrak{g}$ induces a differential

$$
d_{\mathbf{r}^{\sharp}}: \operatorname{Hom}\left(\wedge^{n} \mathfrak{g}^{*}, \mathfrak{g}\right) \rightarrow \operatorname{Hom}\left(\wedge^{n+1} \mathfrak{g}^{*}, \mathfrak{g}\right), d_{\mathbf{r}^{\sharp}}:=l_{2}\left(\mathbf{r}^{\sharp},-\right)-\frac{1}{2} l_{3}\left(\mathbf{r}^{\sharp}, \mathbf{r}^{\sharp},-\right) .
$$

The corresponding cohomology groups are called the cohomology of the $\psi$-twisted triangular $r$-matric $\mathbf{r}$. This cohomology is isomorphic to the Chevalley-Eilenberg cohomology of the Lie algebra $\mathfrak{g}^{*}$ given in (5) with coefficients in $\mathfrak{g}$ given by

$$
\alpha \bar{\bullet} x=\left[\mathbf{r}^{\sharp}(\alpha), x\right]+\mathbf{r}^{\sharp}\left(a d_{x}^{*} \alpha+\psi^{\sharp}\left(x, \mathbf{r}^{\sharp}(\alpha)\right), \quad \text { for } \alpha \in \mathfrak{g}^{*}, x \in \mathfrak{g} .\right.
$$

When $\psi=0$, we get $l_{3}=0$. Therefore, the corresponding cohomology groups are the standard cohomology of the classical triangular $r$-matrix [24].

By the same spirit as of section 4, we may study deformations of a $\psi$-twisted triangular $r$-matrix, their equivalences and rigidity.

\section{$6 \quad$ NS-Lie algebras}

In this section, we introduce a new algebraic structure which is related to twisted Rota-Baxter operators in the same way pre-Lie algebras are related to Rota-Baxter operators. We call such algebras as NS-Lie algebras. Further study on NS-Lie algebras is postponed to a forthcoming paper.

6.1 Definition. An NS-Lie algebra is a vector space $L$ together with bilinear operations $\circ, \curlyvee: L \times L \rightarrow L$ in which $\curlyvee$ is skew-symmetric and satisfying the following two identities

(NS1) $\operatorname{Ass}(x, y, z)-\operatorname{Ass}(y, x, z)+(x \curlyvee y) \circ z=0$,

i.e. $(x \circ y) \circ z-x \circ(y \circ z)-(y \circ x) \circ z+y \circ(x \circ z)+(x \curlyvee y) \circ z=0$,

(NS2) $x \curlyvee(y * z)+c . p .+x \circ(y \curlyvee z)+c . p .=0$,

i.e. $x \curlyvee(y * z)+y \curlyvee(z * x)+z \curlyvee(x * y)+x \circ(y \curlyvee z)+y \circ(z \curlyvee x)+z \circ(x \curlyvee y)=0$,

for all $x, y, z \in L$. Here $x * y=x \circ y-y \circ x+x \curlyvee y$, for $x, y \in L$.

6.2 Remark. If the bilinear operation $\circ$ in the above definition is trivial, one gets that $(L, \curlyvee)$ is a Lie algebra. On the other hand, if $\checkmark$ is trivial, then $(L, \circ)$ becomes a pre-Lie algebra. Thus, NS-Lie algebras are a generalization of both Lie algebras and pre-Lie algebras.

Let us use the following notation in an NS-Lie algebra. For $x \in L$, we consider the map $l_{x}: L \rightarrow L$, $l_{x}(y)=x \circ y$, for $y \in L$. Then the identity (NS1) can be simply written as

$$
\left[l_{x}, l_{y}\right]=l_{x * y},
$$

where on the left-hand side, we use the commutator bracket of linear maps on $L$.

6.3 Proposition. Let $(L, \circ, \curlyvee)$ be an NS-Lie algebra. Then $L$ equipped with the bracket

$$
[x, y]:=x \circ y-y \circ x+x \curlyvee y
$$

is a Lie algebra (called the adjacent Lie algebra of $L$, denoted by $L_{\text {Lie }}$ ). Morever, the Lie algebra $L_{\text {Lie }}$ has a representation on $L$ given by $x \bullet m=x \circ m$, for $x \in L_{\mathrm{Lie}}, m \in L$. 
Proof. For any $x, y, z \in L$, we have

$$
\begin{aligned}
{[x,[y, z]] } & =[x, y \circ z-z \circ y+y \curlyvee z] \\
& =x \circ(y \circ z)-x \circ(z \circ y)+x \circ(y \curlyvee z)-(y \circ z) \circ x+(z \circ y) \circ x-(y \curlyvee z) \circ x+x \curlyvee(y * z) .
\end{aligned}
$$

Similarly,

$$
\begin{aligned}
& {[y,[z, x]]=y \circ(z \circ x)-y \circ(x \circ z)+y \circ(z \curlyvee x)-(z \circ x) \circ y+(x \circ z) \circ y-(z \curlyvee x) \circ y+y \curlyvee(z * x),} \\
& {[z,[x, y]]=z \circ(x \circ y)-z \circ(y \circ x)+z \circ(x \curlyvee y)-(x \circ y) \circ z+(y \circ x) \circ z-(x \curlyvee y) \circ z+z \curlyvee(x * y) .}
\end{aligned}
$$

By adding (23), (24), (25) and using (NS1), we get

$$
\begin{aligned}
{[x,[y, z]]+[y,[z, x]]+[z,[x, y]]=} & -(z \curlyvee y) \circ x-\overline{(x \curlyvee z) \circ y}-\overline{(y) \circ z} \\
& +x \circ(y \curlyvee z)-(y \curlyvee z) \circ x+y \circ(z \curlyvee x)-(z \curlyvee x) \circ y+z \circ(x \curlyvee y)-(x) \circ z \\
& +x \curlyvee(y * z)+y \curlyvee(z * x)+z \curlyvee(x * y) .
\end{aligned}
$$

This is 0 by $(\mathrm{NS} 2)$. Hence $(L,[]$,$) is a Lie algebra.$

The last part follows from the identity (22).

A morphism between NS-Lie algebras is a linear map between underlying vector spaces that commute with structure maps. We denote the category of NS-Lie algebras by NS. The construction of the above proposition yields a functor ()$_{c}: \mathbf{N S} \rightarrow$ Lie from the category of NS-Lie algebras to the category of Lie algebras.

6.4 Proposition. Let $(\mathfrak{g},[]$,$) be a Lie algebra and N: \mathfrak{g} \rightarrow \mathfrak{g}$ be a Nijenhuis operator. Then

$$
x \circ y=[N x, y] \quad \text { and } \quad x \curlyvee y=-N[x, y]
$$

defines an NS-Lie algebra structure on $\mathfrak{g}$.

Proof. For any $x, y, z \in \mathfrak{g}$, we have

$$
\begin{aligned}
& (x \circ y) \circ z-x \circ(y \circ z)-(y \circ x) \circ z+y \circ(x \circ z) \\
& =[N[N x, y], z]-[N x,[N y, z]]-[N[N y, x], z]+[N y,[N x, z]] \\
& =[N[N x, y], z]-[[N x, N y], z]-[N y,[N x, z]]-[N[N y, x], z]+[N y,[N x, z]] \\
& =[N[N x, y], z]-[N[N x, y], z]+[N[N y, x], z]+\left[N^{2}[x, y], z\right]-[N[N y, x], z] \\
& =\left[N^{2}[x, y], z\right]=-[N(x \curlyvee y), z]=-(x \curlyvee y) \circ z .
\end{aligned}
$$

Hence we have proved (NS1). To prove (NS2), we first recall that for a Nijenhuis operator $N$, the deformed bracket

$$
[x, y]_{N}:=x \circ y-y \circ x+x \curlyvee y=[N x, y]-[N y, x]-N[x, y]
$$

is a Lie bracket on $\mathfrak{g}$ and this Lie bracket is compatible with the given Lie bracket [, ] on $\mathfrak{g}$ [13]. In other words, the sum $[]+,[,]_{N}$ is also a Lie bracket, equivalently,

$$
\left[x,[y, z]_{N}\right]+c . p .+[x,[y, z]]_{N}+c . p .=0 .
$$


Thus, we have

$$
\begin{aligned}
x \curlyvee(y * z)+c . p .+x \circ(y \curlyvee z)+c . p . & =-N\left[x,[y, z]_{N}\right]+c . p .-[N x, N[y, z]]+c . p . \\
& =-N\left[x,[y, z]_{N}\right]+c . p .-N[x,[y, z]]_{N}+c . p .=0 .
\end{aligned}
$$

Hence the result is proved.

In [19] Leroux introduced NS-algebras as a generalization of dendriform algebras. We show that NSalgebras give rise to NS-Lie algebras.

6.5 Definition. An (associative) NS-algebra is a vector space $A$ together with three bilinear operations $\prec, \succ, \square: A \otimes A \rightarrow A$ satisfying the following identities

$$
\begin{array}{r}
(x \prec y) \prec z=x \prec(y \circledast z), \quad(x \succ y) \prec z=x \succ(y \prec z), \quad(x \circledast y) \succ z=x \succ(y \succ z), \\
(x \square y) \prec z+(x \circledast y) \square z=x \succ(y \square z)+x \square(y \circledast z), \quad \text { for } x, y, z \in A .
\end{array}
$$

Here $\circledast: A \otimes A \rightarrow A$ is the map defined by $x \circledast y=x \prec y+x \succ y+x \square y$, for $x, y \in A$.

6.6 Proposition. Let $(A, \prec, \succ, \square)$ be an (associative) $N$ S-algebra. Then $(A, \circ, \curlyvee)$ is an NS-Lie algebra, where

$$
x \circ y=x \succ y-y \prec x \quad \text { and } \quad x \curlyvee y=x \square y-y \square x .
$$

Proof. By a direct calculation, we have

$$
\begin{aligned}
\operatorname{Ass}(x, y, z)=(x \circ y) \circ z-x \circ(y \circ z) & =(x \succ y) \succ z-(y \prec x) \succ z-z \prec(x \succ y)+z \prec(y \prec x) \\
& -x \succ(y \succ z)+x \succ(z \prec y)+(y \succ z) \prec x-(z \prec y) \prec x \\
& =-(x \prec y) \succ z-(x \square y) \succ z-(y \prec x) \succ z-z \prec(x \succ y) \\
& +x \succ(z \prec y)+(y \succ z) \prec x-z \prec(y \succ x)-z \prec(y \square x) .
\end{aligned}
$$

Therefore,

$$
\begin{aligned}
\operatorname{Ass}(x, y, z)-\operatorname{Ass}(y, x, z) & =-(x \prec y) \succ z-(x \square y) \succ z-(y \prec x) \succ z-z \prec(x \succ y) \\
& +x \succ(z \prec y)+(y \succ z) \prec x-z \prec(y \succ x)-z \prec(y \square x) \\
& +(y \prec x) \succ z+(y \square x) \succ z+(x \prec y) \succ z+z \prec(y \succ x) \\
& -y \succ(z \prec x)-(x \succ z) \prec y+z \prec(x \succ y)+z \prec(x \square y) \\
& =-(x \square y) \succ z-z \prec(y \square x)+(y \square x) \succ z+z \prec(x \square y) \\
& =-(x \curlyvee y) \succ z+z \prec(x \curlyvee y)=-(x \curlyvee y) \circ z .
\end{aligned}
$$

Thus we have proved (NS1). Next, we have from (27) that

$$
\begin{aligned}
& x \square(y \circledast z)-(x \circledast y) \square z+x \succ(y \square z)-(x \square y) \prec z=0, \\
& y \square(z \circledast x)-(y \circledast z) \square x+y \succ(z \square x)-(y \square z) \prec x=0, \\
& z \square(x \circledast y)-(z \circledast x) \square y+z \succ(x \square y)-(z \square x) \prec y=0 .
\end{aligned}
$$

By adding all these, we get

$$
x \curlyvee(y \circledast z)+y \curlyvee(z \circledast x)+z \curlyvee(x \circledast y)+x \circ(y \square z)+y \circ(z \square x)+z \circ(x \square y)=0 .
$$


Interchanging $y$ and $z$, we get

$$
x \curlyvee(z \circledast y)+z \curlyvee(y \circledast x)+y \curlyvee(x \circledast z)+x \circ(z \square y)+z \circ(y \square x)+y \circ(x \square z)=0 .
$$

Finally, by subtracting (29) from (28), we get (NS2). This completes the proof.

\subsection{Relation with twisted Rota-Baxter operators}

6.7 Proposition. Let $T: M \rightarrow \mathfrak{g}$ be a H-twisted Rota-Baxter operator. Then

$$
u \circ v=T(u) \bullet v \quad \text { and } \quad u \curlyvee v=H(T u, T v), \text { for } u, v \in M,
$$

defines an NS-Lie algebra structure on $M$.

Proof. For any $u, v, w \in M$,

$$
\begin{aligned}
& (u \circ v) \circ w-u \circ(v \circ w)-(v \circ u) \circ w+v \circ(u \circ w) \\
& =T(T(u) \bullet v) \bullet w-T(u) \bullet(T(v) \bullet w)-T(T(v) \bullet u) \bullet w+T(v) \bullet(T(u) \bullet w) \\
& =T(T(u) \bullet v) \bullet w-[T u, T v] \bullet w-T(v) \bullet(T(u) \bullet w)-T(T(v) \bullet u) \bullet w+T(v) \bullet(T(u) \bullet w) \\
& =T(T(u) \bullet v) \bullet w-T(T(u) \bullet v) \bullet w+T(T(v) \bullet u) \bullet w-T H(T u, T v) \bullet w-T(T(v) \bullet u) \bullet w \\
& =-T H(T u, T v) \bullet w=-(u \curlyvee v) \circ w .
\end{aligned}
$$

Thus the condition (NS1) holds. For (NS2), we observe that

$$
\begin{aligned}
& u \curlyvee(v * w)+c . p .+u \circ(v \curlyvee w)+c . p . \\
& =H(T u, T(v * w))+c . p .+T(u) \bullet H(T v, T w)+c . p . \\
& =H(T u,[T v, T w])+c . p .+T(u) \bullet H(T v, T w)+c . p .=0 \quad(\text { as } H \text { is a 2-cocycle }) .
\end{aligned}
$$

Hence $(M, \circ, \curlyvee)$ is an NS-Lie algebra.

6.8 Remark. Let $N: \mathfrak{g} \rightarrow \mathfrak{g}$ be a Nijenhuis operator on a Lie algebra $(\mathfrak{g},[]$,$) . It follows from Example$ 2.9 and Proposition 6.7 that the vector space $\mathfrak{g}$ carries an NS-Lie algebra structure $(\mathfrak{g}, \diamond, \curlyvee)$ given by

$$
x \diamond y=\operatorname{id}(x) \bullet y=x \bullet y=[N x, y] \quad \text { and } \quad x \curlyvee y=H(\operatorname{id}(x), \operatorname{id}(y))=H(x, y)=-N[x, y] .
$$

This NS-Lie algebra structure on $\mathfrak{g}$ is precisely the one obtained in Proposition 6.4.

Let $T: M \rightarrow \mathfrak{g}$ be a $H$-twisted Rota-Baxter operator. Suppose $\mathfrak{g}^{\prime}$ is another Lie algebra, $M^{\prime}$ is a $\mathfrak{g}^{\prime}$-module and $H^{\prime} \in C_{\mathrm{CE}}^{2}\left(\mathfrak{g}^{\prime}, M^{\prime}\right)$ is a 2-cocycle. Let $T^{\prime}: M^{\prime} \rightarrow \mathfrak{g}^{\prime}$ be a $H^{\prime}$-twisted Rota-Baxter operator. A weak morphism from $T$ to $T^{\prime}$ consist of linear maps $\phi: \mathfrak{g} \rightarrow \mathfrak{g}^{\prime}$ and $\psi: M \rightarrow M^{\prime}$ satisfying $\psi(x \bullet u)=$ $\phi(x) \bullet \psi(u), \psi \circ H=H^{\prime} \circ(\phi \otimes \phi)$ and $\phi \circ T=T^{\prime} \circ \psi$, for $x \in \mathfrak{g}$ and $u \in M$. Thus, a morphism of twisted Rota-Baxter operators in the sense of Definition 2.5 is a weak morphism $(\phi, \psi)$ in which $\phi$ is a Lie algebra morphism.

Let TRB denotes the category of twisted Rota-Baxter operators and weak morphisms between them. In the previous proposition, we construct an NS-Lie algebra from a twisted Rota-Baxter operator. In fact, this construction gives rise to a functor $F: \mathbf{T R B} \rightarrow \mathbf{N S}$.

Let $(L, \circ, \curlyvee)$ be an NS-Lie algebra with the adjacent Lie algebra $L_{\text {Lie }}=(L,[]$,$) . Then it has been$ shown that the Lie algebra $L_{\text {Lie }}$ has a (well-defined) representation on $L$ given by

$$
x \bullet u:=x \circ u, \text { for } x \in L_{\text {Lie }}, u \in L .
$$


With this notation, the map $H: L_{\mathrm{Lie}} \times L_{\mathrm{Lie}} \rightarrow L, H(x, y)=x \curlyvee y$ is a 2-cocycle in the Chevalley-Eilenberg cohomology of the Lie algebra $L_{\mathrm{Lie}}$ with coefficients in the representation $L$. It is easy to see that the condition (NS2) is equivalent to the fact that $H$ is a 2-cocycle. The identity map $1: L \rightarrow L_{\text {Lie }}$ is then a $H$-twisted Rota-Baxter operator. This construction of a twisted Rota-Baxter operator from an NS-Lie algebra is also functorial and defines a functor $G: \mathbf{N S} \rightarrow$ TRB. Moreover, we get an adjoint relation

$$
\operatorname{Hom}_{\mathbf{N S}}(L, F(T)) \cong \operatorname{Hom}_{\mathbf{T R B}}(G(L), T),
$$

where $T$ is a $H$-twisted Rota-Baxter operator and $L$ is any NS-Lie algebra.

\section{Twisted generalized complex structures}

In this section, we introduce twisted generalized complex structures on modules over a Lie algebra. When one considers the coadjoint module $\mathfrak{g}^{*}$ of a Lie algebra $\mathfrak{g}$, one gets twisted generalized complex structures on $\mathfrak{g}$.

Let $\mathfrak{g}$ be a Lie algebra, $M$ a $\mathfrak{g}$-module and $H \in C_{\mathrm{CE}}^{2}(\mathfrak{g}, M)$ be a 2-cocycle. Consider the semi-direct product Lie algebra $\mathfrak{g} \ltimes_{H} M$ with the bracket given in (2). Any linear map $J: \mathfrak{g} \oplus M \rightarrow \mathfrak{g} \oplus M$ must be of the form

$$
J=\left(\begin{array}{cc}
N & T \\
\sigma & -S
\end{array}\right)
$$

for some linear maps $N \in \operatorname{End}(\mathfrak{g}), S \in \operatorname{End}(M), T: M \rightarrow \mathfrak{g}$ and $\sigma: \mathfrak{g} \rightarrow M$. These maps are called the structure components of $J$. The reason behind considering the linear map as $-S$ (instead of $S$ ) will be clear from Proposition 7.11.

7.1 Definition. An $H$-twisted generalized complex structure on $M$ over the Lie algebra $\mathfrak{g}$ is a linear map $J: \mathfrak{g} \oplus M \rightarrow \mathfrak{g} \oplus M$ that satisfies the following conditions

(i) $J$ is almost complex: $J^{2}=-$ id,

(ii) integrability condition: $[J r, J s]-[r, s]-J([J r, s]+[r, J s])=0$, for $r, s \in \mathfrak{g} \oplus M$.

Note that we have used the $H$-twisted semi-direct product bracket (2) in the above integrability condition. A generalized complex structure on $M$ over the Lie algebra $\mathfrak{g}$ is a $H$-twisted generalized complex structure for $H=0$.

In [5] Crainic gives a characterization of generalized complex manifolds. A similar theorem in the context of $H$-twisted generalized complex structures is the following.

7.2 Theorem. A linear map $J: \mathfrak{g} \oplus M \rightarrow \mathfrak{g} \oplus M$ of the form (30) is a $H$-twisted generalized complex 
structure on $M$ over the Lie algebra $\mathfrak{g}$ if and only if its structure components satisfy the following identities:

$$
\begin{aligned}
& N T=T S, \\
& N^{2}+T \sigma=-\mathrm{id}, \\
& S \sigma=\sigma N, \\
& S^{2}+\sigma T=-\mathrm{id}, \\
& {[T u, T v]=T(T u \bullet v-T v \bullet u),} \\
& T u \bullet S v-T v \bullet S u-H(T u, T v)=S(T u \bullet v-T v \bullet u), \\
& {[N x, T u]-N[x, T u]=T(N x \bullet u-x \bullet S u+H(x, T u)),} \\
& \sigma[T u, x]-T u \bullet \sigma x-H(T u, N x)=x \bullet u+N x \bullet S u-S(N x \bullet u-x \bullet S u+H(x, T u)), \\
& {[N x, N y]-[x, y]-N([N x, y]+[x, N y])=T(x \bullet \sigma y-y \bullet \sigma x+H(x, N y)-H(y, N x)),} \\
& N x \bullet \sigma y-N y \bullet \sigma x+H(N x, N y)-H(x, y)-\sigma([N x, y]+[x, N y])= \\
& \quad-S(x \bullet \sigma y-y \bullet \sigma x+H(x, N y)-H(y, N x)),
\end{aligned}
$$

for all $x, y \in \mathfrak{g}$ and $u, v \in M$.

Proof. The condition $J^{2}=-$ id is same as

$$
\left(\begin{array}{c}
N^{2}(x)+N T(u)+T \sigma(x)-T S(u) \\
\sigma N(x)+\sigma T(u)-S \sigma(x)+S^{2}(u)
\end{array}\right)=\left(\begin{array}{c}
-x \\
-u
\end{array}\right)
$$

which is equivalent to the identities (31)-(34). Next consider the integrability condition of $J$. For $r=$ $u, s=v \in M$, the integrability criteria is equivalent to (35) and (36). For $r=x \in \mathfrak{g}$ and $s=u \in M$, the integrability is equivalent to (37) and (38). Finally, for $r=x, s=y \in \mathfrak{g}$, we get the identities (39) and (40).

7.3 Remark. Note that the condition (35) implies that the map $T: M \rightarrow \mathfrak{g}$ is a Rota-Baxter operator. Moreover, the condition (36) is equivalent to the fact that

$$
\operatorname{Gr}((T, S))=\{(T u, S u) \mid u \in M\} \subset \mathfrak{g} \oplus M
$$

is a subalgebra of the $(-H)$-twisted semi-direct product $\mathfrak{g} \ltimes_{-H} M$.

The above theorem ensures the following examples of (twisted) generalized complex structures on modules over Lie algebras.

7.4 Example. (Opposite g.c.s.) Let $J=\left(\begin{array}{cc}N & T \\ \sigma & -S\end{array}\right)$ be a $H$-twisted generalized complex structure on $M$ over $\mathfrak{g}$. Then $\bar{J}=\left(\begin{array}{cc}N & -T \\ -\sigma & -S\end{array}\right)$ is a $(-H)$-twisted generalized complex structure, called the opposite of $J$.

7.5 Example. Let $T: M \rightarrow \mathfrak{g}$ be an invertible Rota-Baxter operator. Then $J=\left(\begin{array}{cc}0 & T \\ -T^{-1} & 0\end{array}\right)$ is a generalized complex structure on $M$ over $\mathfrak{g}(H=0)$.

Complex structures on modules over Lie algebra also lie in generalized complex structures. We start with the following known definition.

7.6 Definition. A complex structure on a Lie algebra $\mathfrak{g}$ is a linear map $I: \mathfrak{g} \rightarrow \mathfrak{g}$ satisfying $I^{2}=-\mathrm{id}$ and

$$
[I x, I y]-[x, y]-I([I x, y]+[x, I y])=0, \text { for } x, y \in \mathfrak{g} .
$$


7.7 Definition. Let $\mathfrak{g}$ be a Lie algebra and $M$ be a $\mathfrak{g}$-module. A complex structure on $M$ over the Lie algebra $\mathfrak{g}$ is a pair $\left(I, I_{M}\right)$ of linear maps $I \in \operatorname{End}(\mathfrak{g})$ and $I_{M} \in \operatorname{End}(M)$ satisfying the followings

$\triangleright I$ is a complex structure on $\mathfrak{g}$,

$\triangleright I_{M}^{2}=-\mathrm{id}$ and

$$
I(x) \bullet I_{M}(u)-x \bullet u-I_{M}\left(I(x) \bullet u+x \bullet I_{M}(u)\right)=0, \text { for } x \in \mathfrak{g}, u \in M .
$$

7.8 Proposition. A pair $\left(I, I_{M}\right)$ is a complex structure on $M$ over the Lie algebra $\mathfrak{g}$ if and only if $I \oplus I_{M}: \mathfrak{g} \oplus M \rightarrow \mathfrak{g} \oplus M$ is a complex structure on the semi-direct product Lie algebra $\mathfrak{g} \ltimes M$.

Proof. The linear map $I \oplus I_{M}$ is a complex structure on the semi-direct product $\mathfrak{g} \ltimes M$ if and only if $\left(I \oplus I_{M}\right)^{2}=-\mathrm{id}$ (equivalently, $I^{2}=-\mathrm{id}$ and $\left.I_{M}^{2}=-\mathrm{id}\right)$ and

$$
\left[\left(I x, I_{M} u\right),\left(I y, I_{M} v\right)\right]-[(x, u),(y, v)]-\left(I \oplus I_{M}\right)\left(\left[\left(I x, I_{M} u\right),(y, v)\right]+\left[(x, u),\left(I y, I_{M} v\right)\right]\right)=0
$$

or, equivalently,

$$
\begin{aligned}
([I x, I y]-[x, y]-I([I x, y]+[x, I y]), & I x \bullet I_{M} v-I y \bullet I_{M} v-(x \bullet v-y \bullet u) \\
& \left.-I_{M}\left(I x \bullet v-y \bullet I_{M} u+x \bullet I_{M} v-I y \bullet u\right)\right)=0 .
\end{aligned}
$$

The last identity is equivalent to the fact that $I$ is a complex structure on $\mathfrak{g}$ and the identity (41) holds. In other words $\left(I, I_{M}\right)$ is a complex structure on $M$ over the Lie algebra $\mathfrak{g}$.

7.9 Example. Let $\left(I, I_{M}\right)$ be a complex structure on $M$ over the Lie algebra $\mathfrak{g}$. Then $J=\left(\begin{array}{cc}I & 0 \\ 0 & I_{M}\end{array}\right)$ is a generalized complex structure on $M$ over $\mathfrak{g}$. Note that here $S=-I_{M}$.

Next, we consider twisted generalized complex structures on Lie algebras and show that they are related to twisted generalized complex structures on coadjoint modules. Let $\mathfrak{g}$ be a Lie algebra and $\psi \in \wedge^{3} \mathfrak{g}^{*}$ be a 3 -cocycle in the cohomology of $\mathfrak{g}$ with coefficients in $\mathbb{K}$. Consider the coadjoint representation of $\mathfrak{g}$ on $\mathfrak{g}^{*}$. Then $\psi^{\sharp}: \wedge^{2} \mathfrak{g} \rightarrow \mathfrak{g}^{*}$ is a 2 -cocycle in the cohomology of $\mathfrak{g}$ with coefficients in $\mathfrak{g}^{*}$. The corresponding $\psi^{\sharp}$-twisted semi-direct product algebra $\mathfrak{g} \ltimes_{\psi^{\sharp}} \mathfrak{g}^{*}$ is given by the bracket

$$
[(x, \alpha),(y, \beta)]=\left([x, y], \operatorname{ad}_{x}^{*} \beta-\operatorname{ad}_{y}^{*} \alpha+\psi^{\sharp}(x, y)\right) .
$$

The direct sum $\mathfrak{g} \oplus \mathfrak{g}^{*}$ also carries a non-degenerate inner product given by $\langle(x, \alpha),(y, \beta)\rangle=\frac{1}{2}(\alpha(y)+\beta(x))$.

7.10 Definition. A $\psi$-twisted generalized complex structure on $\mathfrak{g}$ consists of a linear map $J: \mathfrak{g} \oplus \mathfrak{g}^{*} \rightarrow \mathfrak{g} \oplus \mathfrak{g}^{*}$ satisfying

(0) orthogonality: $\langle J r, J s\rangle=\langle r, s\rangle$,

(i) almost complex: $J^{2}=-$ id,

(ii) integrability: $\quad[J r, J s]-[r, s]-J([J r, s]+[r, J s])=0$, for $r, s \in \mathfrak{g} \oplus \mathfrak{g}^{*}$.

Note that the orthogonality condition (0) implies that $J$ must be of the form

$$
J=\left(\begin{array}{cc}
N & \mathbf{r}^{\sharp} \\
\sigma_{b} & -N^{*}
\end{array}\right),
$$

for some $N \in \operatorname{End}(\mathfrak{g}), \mathbf{r} \in \wedge^{2} \mathfrak{g}$ and $\sigma \in \wedge^{2} \mathfrak{g}^{*}$. However, the conditions (i) and (ii) of the definition imposes some relations between structure components of $J$.

Thus, a $\psi$-twisted generalized complex structure on $\mathfrak{g}$ can also be considered as a triple $(N, \mathbf{r}, \sigma)$ such that the linear map $J$ of the form (42) is almost complex and satisfies the integrability condition. 
7.11 Proposition. Let $\mathfrak{g}$ be a Lie algebra and $\psi \in \wedge^{3} \mathfrak{g}^{*}$ be a 3-cocycle in the cohomology of $\mathfrak{g}$ with coefficients in $\mathbb{K}$. A triple $(N, \mathbf{r}, \sigma)$ is a $\psi$-twisted generalized complex structure on $\mathfrak{g}$ if and only if the linear map $J=\left(\begin{array}{cc}N & \mathbf{r}^{\sharp} \\ \sigma_{b} & -N^{*}\end{array}\right): \mathfrak{g} \oplus \mathfrak{g}^{*} \rightarrow \mathfrak{g} \oplus \mathfrak{g}^{*}$ is a $\psi^{\sharp}$-twisted generalized complex structure on the coadjoint module $\mathfrak{g}^{*}$ over the Lie algebra $\mathfrak{g}$.

Acknowledgements. The author thanks the referee for his/her valuable advice which improved the paper. The research is supported by the fellowship of Indian Institute of Technology (IIT) Kanpur.

Data Availability Statement. Data sharing is not applicable to this article as no new data were created or analyzed in this study.

\section{References}

[1] G. Baxter, An analytic problem whose solution follows from a simple algebraic identity, Pacific J. Math. 10 (1960), 731-742.

[2] P. Cartier, On the structure of free Baxter algebras, Advances in Math. 9 (1972), 253-265.

[3] A. Connes and D. Kreimer, Renormalization in quantum field theory and the Riemann-Hilbert problem. I. The Hopf algebra structure of graphs and the main theorem, Comm. Math. Phys. 210 (2000), no. 1, 249-273.

[4] T. J. Courant, Dirac manifolds, Trans. Amer. Math. Soc. 319 (1990), no. 2, 631-661.

[5] M. Crainic, Generalized complex structures and Lie brackets, Bull. Braz. Math. Soc. (N.S.) 42 (2011), no. 4, 559-578.

[6] A. Das, Deformations of associative Rota-Baxter operators, J. Algebra 560 (2020) 144-180.

[7] Y. Frégier and M. Zambon, Simultaneous deformations and Poisson geometry, Compositio Mathematica, 151(9), 1763-1790.

[8] M. Gerstenhaber, On the deformation of rings and algebras, Ann. of Math. (2) 79 (1964), 59-103.

[9] E. Getzler, Lie theory for nilpotent $L_{\infty}$-algebras, Ann. of Math. (2) 170 (2009), no. 1, 271-301.

[10] M. Gualtieri, Generalized complex geometry, Oxford University D.Phil. thesis, 107 pages, arXiv:math/0401221 (math).

[11] N. Hitchin, Generalized Calabi-Yau manifolds, Q. J. Math. 54 (2003), no. 3, 281-308.

[12] C. Klimčík and T. Strobl, WZW-Poisson manifolds, J. Geom. Phys. 43 (2002), 341-344.

[13] Y. Kosmann-Schwarzbach and F. Magri, Poisson-Nijenhuis structures, Ann. Inst. H. Poincaré Phys. Théor. 53 (1990), no. 1, 35-81.

[14] Y. Kosmann-Schwarzbach and M. Yakimov, Modular classes of regular twisted Poisson structures on Lie algebroids, Lett. Math. Phys. 80 (2007), 183-197.

[15] B. A. Kupershmidt, What a classical r-matrix really is, J. Nonlinear Math. Phys. 6 (1999), no. 4, 448-488. 
[16] T. Lada and M. Markl, Strongly homotopy Lie algebras, Comm. Algebra 23 (1995), no. 6, $2147-2161$.

[17] T. Lada and J. Stasheff, Introduction to SH Lie algebras for Physicists, Internat. J. Theoret. Phys. 32 (1993), no. 7, 1087-1103.

[18] A. Lazarev, Y. Sheng and R. Tang, Deformations and homotopy theory of relative Rota-Baxter Lie algebras, Comm. Math. Phys. 383 (2021), 595-631.

[19] P. Leroux, Construction of Nijenhuis operators and dendriform trialgebras, Int. J. Math. Math. Sci. 49 (2004) 2595-2615.

[20] D. Manchon, A short survey on pre-Lie algebras, Noncommutative geometry and Physics: renormalization, motives, index theory, ESI Lect. Math. Phys., Eur. Math. Soc., Zürich, 2011, pp. 89-102.

[21] M. Markl, Intrinsic brackets and the $L_{\infty}$-deformation theory of bialgebras, J. Homotopy Relat. Struct. 5(1) (2010) 177-212.

[22] G.-C. Rota, Baxter algebras and combinatorial identities, I, II, Bull. Amer. Math. Soc. 75 (1969), 325-329; ibid. 751969 330-334.

[23] P. Ševera and A. Weinstein, Poisson geometry with a 3-form background, Progr. Theoret. Phys. Suppl. 144 (2001), 145-154.

[24] R. Tang, C. Bai, L. Guo and Y. Sheng, Deformations and their controlling cohomologies of O-operators, Comm. Math. Phys. 368 (2019), no. 2, 665-700.

[25] K. Uchino, Quantum analogy of Poisson geometry, related dendriform algebras and Rota-Baxter operators, Lett. Math. Phys. 85 (2008), no. 2-3, 91-109.

[26] Th. Voronov, Higher derived brackets and homotopy algebras, J. Pure Appl. Algebra 202 (2005), no. $1-3,133-153$.

[27] T. Zhang, X. Gao and L. Guo, Reynolds operators and their free objects from bracketed words and rootes trees, J. Pure Appl. Algebra 225 (2021), no. 12, 106766. 